\title{
DISTRIBUTED STREAMING FOR VIDEO ON DEMAND
}

by

\section{SHRADDHA RUMADE}

Presented to the Faculty of the Graduate School of

The University of Texas at Arlington in Partial Fulfillment

of the Requirements

for the Degree of

MASTER OF SCIENCE IN COMPUTER SCIENCE \& ENGINEERING

THE UNIVERSITY OF TEXAS AT ARLINGTON

August 2005 


\section{ACKNOWLEDGEMENTS}

I would like to express my earnest appreciation to my supervising professor, Dr. Ramesh Yerraballi, for his guidance and incessant patience that encouraged me to conduct this work. His expert advice throughout my Masters has greatly assisted me in completing this thesis. I would also like to thank Dr. Gautam Das and Dr. Gergely Záruba for being on my committee and reviewing my work.

I feel a deep sense of gratitude towards my father who has been my greatest inspiration through all the endeavors I have undertaken. To complete this chain of recognition I would like to thank my friends for their support.

July 21, 2005 


\section{ABSTRACT \\ DISTRIBUTED STREAMING FOR \\ VIDEO ON DEMAND}

Publication No.

Shraddha Rumade, MS. CSE

The University of Texas at Arlington, 2005

Supervising Professor: Dr. Ramesh Yerraballi

With the advent of digital media, implementing reliable Video on Demand (VoD) systems over the best effort Internet is a potential issue. Distributed streaming for Video on Demand presents a combination of two techniques. The first being Distributed Video Streaming using Multicast (DVSM) [1] involves video streaming from multiple servers to overcome path congestion. The second technique, Asynchronous Hybrid mechanism for Video on Demand [2] implements segmentationbased periodic broadcast to effectively share network bandwidth. In this work we aim at providing path diversity of multiple senders and maximizing bandwidth sharing via multicast. We exploit alternate paths in case of congestion on a path while streaming 
segments of video. Distributed Streaming for Video on Demand uses similar algorithms as in DVSM and extends them to incorporate the segmentation-based periodic broadcast technique in Asynchronous VoD. Due to segmentation-based multicast and patching the system can provide low access latencies for requests arriving in an ad hoc manner.

This work introduces a robust Distributed Video on Demand Architecture by proposing modifications to the algorithms and mathematical relations in the base techniques. 


\section{TABLE OF CONTENTS}

ACKNOWLEDGEMENTS.................................................................... ii

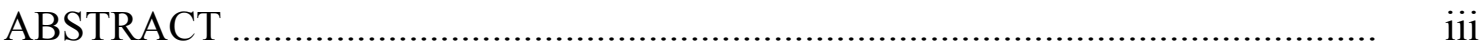

LIST OF ILLUSTRATIONS ............................................................... viii

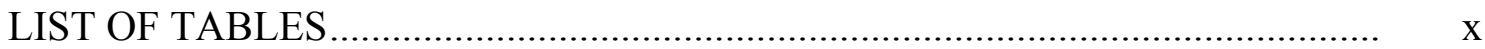

Chapter

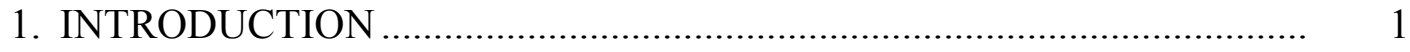

1.1 Motivation ......................................................................... 3

1.2 Related Work ............................................................................ 3

1.2.1 Two Hybrid Multicast Algorithms for Distributed VoD Systems .................................................................. 4

1.2.2 A Distributed VOD Server Based on VIA and Interval Cache ...................................................................................... 5

1.2.3 Selecting among Replicated Batching Video-on-Demand Servers ........................................................................ 6

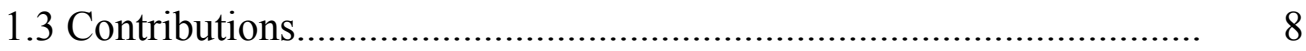

1.4 Thesis Organization .................................................................. 9

2. BACKGROUND ....................................................................... $\quad 10$

2.1 Distributed Video Streaming using Multicast................................. 10

2.1.1 Introduction............................................................... 10

2.1.2 Bandwidth Estimation .......................................................... 11 
2.1.3 Rate Allocation ................................................................... 13

2.1.4 Packet Partitioning and Synchronization Sequence Number ... 14

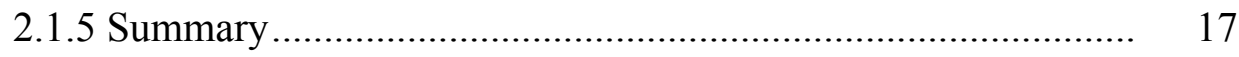

2.2 A New Asynchronous Hybrid Mechanism for Video on Demand ..... 18

2.2.1 Introduction ..................................................................... 18

2.2.2 Assumptions ............................................................. 18

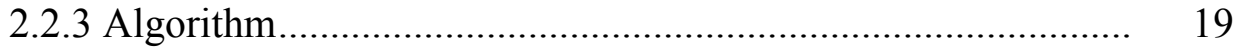

2.2.4 Access Latency for a User Request .................................... 22

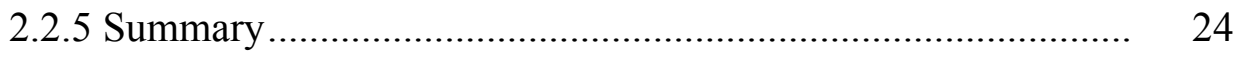

3. PROBLEM STATEMENT …............................................................. 25

4. DISTRIBUTED STREAMING FOR VIDEO ON DEMAND ................... 27

4.1 System Model .......................................................................... 27

4.2 Multicast Schedule on Senders .................................................. 31

4.3 Bandwidth Estimation \& Sending Rates....................................... 32

4.4 Control Packets and Synchronization Sequence Number................. 33

4.5 Segment Partitioning Algorithm (SPA) ...................................... 35

4.6 Playback Continuity............................................................... 36

4.7 Buffer Space Requirement......................................................... 37

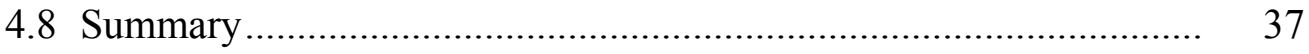

5. SIMULATION STUDY ............................................................... 38

5.1 Model ................................................................................... 38

5.2 Simulation Scenario \& Parameters .............................................. 40 


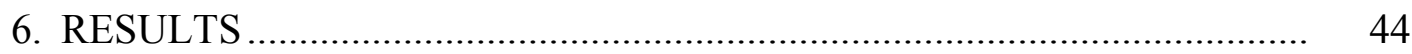

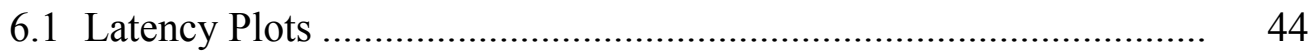

6.2 Network Characteristic Plots ............................................................ 47

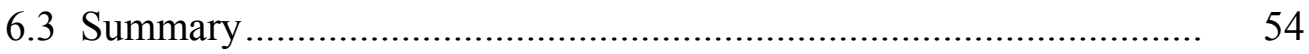

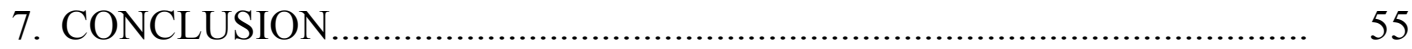

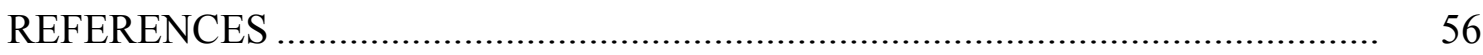

BIOGRAPHICAL INFORMATION............................................................. 58 


\section{LIST OF ILLUSTRATIONS}

Figure $\quad$ Page

2.1 A Distributed Video Streaming with Multicast Example ............................ 11

2.2 Bandwidth Estimation for each Sender................................................... 12

$2.3 \quad$ Rate Allocation Example ...................................................................... 13

2.4 Example of receiving SSN in differing order....................................... 14

2.5 Channels: Partitioning and Bandwidth................................................ 20

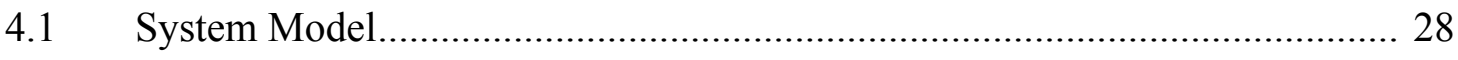

4.2 Channel-Partitioning on Central Server .............................................. 29

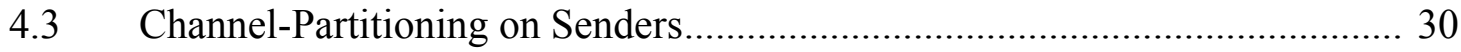

4.4 Initial Segment Schedule on Senders ................................................. 32

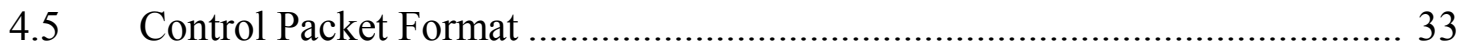

5.1 NSFNet Backbone Network for Simulation ....................................... 40

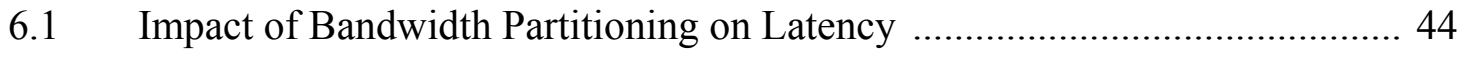

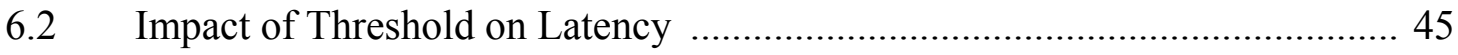

6.3 Latency when supporting more Videos ............................................. 46

6.4 Impact of Increasing the Net Bandwidth .......................................... 47

6.5 Loss rates of each sender measured at R7 ......................................... 48

6.6 Throughputs of S1 and S2 measured at R7 (1) .................................... 49

6.7 Throughputs of S1 and S2 measured at R7 (2) ...................................... 49 
6.8 Loss rates of each sender measured at R1 ............................................. 50

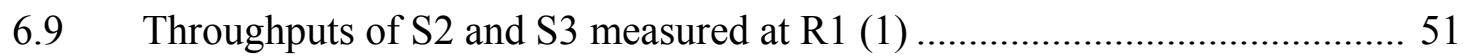

6.10 Throughputs of S2 and S3 measured at R1 (2) ........................................ 51

6.11 Loss rates of S4 and S5 measured at R1 ............................................. 52

6.12 Throughputs of S4 and S5 measured at R1 (1) ........................................ 53

6.13 Throughputs of S4 and S5 measured at R1 (2) ..................................... 53

6.14 Latency: 6 Servers with Selective Placement ......................................... 54 


\section{LIST OF TABLES}

Table

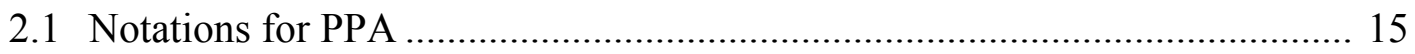

2.2 Terminology used in the VoD Model ..................................................... 19

2.3 Simulation Parameters (Scenario 2) …….................................................... 42 


\section{CHAPTER 1}

\section{INTRODUCTION}

Implementing Video on Demand systems efficiently over the Internet has been a poser due to its high data rate demands and loss and delay sensitivity of media. Video on demand $(\mathrm{VoD})$ systems allow users to choose from a set of videos to play as well as when to play the selected video. The users interact with the server for playback, pause and forward-reverse functions. The basic components of a generic VoD system are users, service providers, program providers and network. The users request videos from the service providers which in turn get the video from program providers and make it available to the clients via the network. A single program provider or server might lead to larger access latency for clients. Thus we need to distribute the content on a group of linked VoD servers for shorter access times. Distributed VoD servers such as the Yima [3] and VoDKA [4] provide a cost effective solution for Video on Demand services.

Streaming video is not a trivial task with many difficulties. They require relatively high bandwidths while to enable streaming video over standard DSL connections it is desirable to limit the bandwidth used. Thus we need better compression techniques. Streaming video requires "real-time" transfers to avoid interruptions in the playback process. This requires the video servers to be able to continuously stream the video and avoid network congestion. To address this issue, 
standards are now available to reserve bandwidth resources along the network. Multicasting may also be used to further reduce network bandwidth requirements.[14]

Since streaming video is usually "bursty" the video clients have a receive buffer to store out of sync or the following part of the stream. But the buffers size is limited giving rise to overflow or underflow if the transmitted bit rate is not smooth enough. To address this issue, additional protocols are used to manage the timing issues. Although adequate for low bit rate applications, systems using bit rates greater than about $1 \mathrm{Mbps}$ still require "tuning" to achieve optimum and reliable performance.

Most of the research in Video on Demand systems focuses on selecting among replicated servers to serve a client request completely i.e. once a request is directed to a server that server takes care of the entire video transmission to the client or a multicast group of clients. In this work we aim at providing the single video content from different servers in an interleaved fashion such that the system can overcome path congestion as well as provide continuous streaming of video to avoid break in the video playback. Distributed Video Streaming using multicast (DVSM) proposes a protocol that provides such path diversity. The Asynchronous Hybrid mechanism for VoD introduces segmentation-based broadcasting and patching which provide low user waiting times for requests arriving in an ad hoc manner.

This thesis presents a combination of DVSM and Asynchronous Hybrid mechanism for VoD. The distributed server architecture in DVSM is modified to suit the VoD model by using segmentation-based multicast that ensures low access latency and smooth playback of video. 


\section{$\underline{1.1 \text { Motivation }}$}

The main aim of this work is to devise a new strategy for Video on Demand that is robust against congestion, efficient with respect to bandwidth utilization and provides low access latency to users.

DVSM is a multicast protocol for streaming video to multiple receivers from a set of distributed servers. The distributed server architecture provides path diversity in case of congestion while multicasting increases the efficiency and scalability of the protocol. Although DVSM proves to be an efficient protocol for VoD systems, alone by itself it can not provide true Video on Demand characteristics. So we combine DVSM with another Video on Demand strategy viz. Asynchronous hybrid mechanism for Video on Demand. We need to take care of the issue that user requests can arrive at any time and request for any video from a set of available ones. A periodic schedule for the video segments that are multicast from different servers should be determined such that any request that arrives is guaranteed a minimum delay. This work aims at addressing all of these and similar issues. Implementing DVSM for VoD can ensure a wider deployment of the true VoD system.

\subsection{Related Work}

In this sub-chapter we cover some of the relevant research work on distributed VoD systems. 


\subsubsection{Two Hybrid Multicast Algorithms for Distributed VoD Systems [5]}

Batching and patching are two commonly used techniques for multicast approaches. This research combines the Maximum Factored Queue Length (MFQL) batching scheme and a Threshold-based patching scheme to propose two hybrid multicast algorithms. The basic idea is to link up small VoD servers to a network such that servers with idle retrieval bandwidth can help servers that are temporarily overloaded.

The videos are required on each server according to their popularity which follows a Zipf law distribution. Batching basically means queuing requests for a certain amount of time and then using a single stream to server them. The Maximum Factored Queue Length (MFQL) scheme schedules the videos with the maximum factored queue length. A weighting factor is applied to each video queue length where the weight is inversely proportional to the popularity of the video. A patching policy reduces the server and network bandwidth usage without introducing any client startup delay. Threshold-based patching introduces a threshold to decide the frequency at which a new stream is initiated.

This research proposes two algorithms namely Local Threshold (LTH) and Remote and Local Threshold (RLTH). Every server maintains a queue per video. When there are multiple video queues in a server and a slot resource is available the MFQL algorithm is used to decide which video queue is best to schedule at that time. If the scheduled video is local and there is sufficient local bandwidth, all the pending requests in the queue of the selected video are served using a single multicast. All the requests 
that arrive within the threshold of the existing complete stream of the video are served using a partial stream. The others are queued until the next multicast stream of the video.

For remote requests in the LTH algorithm the scheduled remote requests queue is served if network resources are available. All other requests that arrive at the remote server are queued. In the RLTH algorithm when a remote requests queue is scheduled at a server and there are available resources in the server and the network, then the server notifies the other servers the following information: 1) a remote stream for the requested video is going to be initiated; 2) the identifier number of the server that carries on the service; 3) threshold for the video. The remaining servers join the multicast stream if they have pending requests for that video. If a new request arrives at a server in this multicast group and it lies within the threshold notified in the beginning then it is serviced with a remote partial stream from the multicast server.

The key issue remains to deciding on an efficient threshold. The main drawback is that it has no alternative in case of path congestion.

\subsubsection{A Distributed VOD Server Based on VIA and Interval Cache}

[6] adopts Virtual Interface Architecture (VIA) communication protocol and the interval cache algorithm to minimize the time required to transfer data across the connecting network. TCP/IP a representative communication protocol has many software overheads. Hence this work suggests a distributed VoD server that uses the communication protocol which allows maximum bandwidth utilization. The interval cache algorithm minimizes the load on the interconnection network. 
VIA is a user-level communication protocol for PC cluster systems where the context switching overhead is removed by performing the communications tasks at the user level instead of the kernel level. Thus VIA allows the user software to fully utilize the physical bandwidth of the network. VIA uses Virtual Interfaces (VIs) to reduce the communication overhead. A VI functions as a communication endpoint for a node and as a communication channel between two nodes. VI User Agent provides routines for data transfer, connection and queue management, memory registration and error handling.

A video cache located in the main memory of each server node stores video data transferred from remote disks using the interval cache algorithm. When there are consecutive client requests for the same video, the video data corresponding to the time gap between these requests is cached. The video data with shorter time gaps between client requests are considered as more popular and are assigned higher priority. This high priority data is then first cached on the video cache and the hit rate is improved.

Although the performance of the system is improved with VIA and the interval cache, there are added overheads of VIs and their functionality.

\subsubsection{Selecting among Replicated Batching Video-on-Demand Servers [7]}

Designers of VoD systems strive to achieve low access latency for customers. One approach is to batch clients requesting the same video and to serve clients in the same batch with one multicast video stream. It has the advantage that it can save server resources as well as server access and network bandwidth. Thus allows the scalability without sacrificing access latency. VoD server replication is another approach that can 
allow a VoD service to handle a large number of clients. But it needs to be coupled with appropriate selection techniques in order to make efficient use of the increased capacity. This work investigates the design of server selection techniques for a system of replicated batching VoD servers.

A large number of clients make requests to the VoD system and this work proposes 6 algorithms on how to best direct a client's request to one of the replicated servers. The first option, the Closest-server-first algorithm, selects the server which is closest to the client using the network hop-count measure. The Optimized closestserverfirst algorithm selects the closest server among those with free channels. If no servers have free channels, the closest server is selected.

The Register-all algorithm will put the client request into the corresponding queue at all of the video servers. When the client request is satisfied at any one server, the request is withdrawn from the other server queues. As an alternative, the Maximum$M F Q$-rank-first algorithm computes the destination queue rank at each server and sends the client request to the queue with the best MFQ rank. That is, best MFQ rank is used as a heuristic to select a server that will reduce the latency to serve the client request.

The Minimum Expected Cost makes use of the number of free channels per server, the hop count to each server, and the MFQ value at each server. Shorter access latency and less bandwidth consumption lead to a smaller expected cost. The expected cost measure is a weighted sum of three terms, and the client selects the server with smallest expected cost. The last algorithm in this work, Merging-Aware Minimum Expected Cost (MAMEC) is used in case of patching. It considers an additional term to 
the server cost that reflects the preference for a server that can start a patching channel soon. Thus with the exception of the very naive Closest Server selection technique, server replication can be used to increase the capacity of the service leading to improved performance.

All of these works concentrate on providing an efficient way of selecting one amongst the distributed servers and streaming the video with minimum delay. But the issue of delay due to congestion during streaming has not been considered. Due to path congestion the receiver may not be able to receive the video even though he started receiving it with a very small waiting time. This research therefore aims at providing viable solutions to issues arising due to delay especially caused while a video is being streamed to the receiver(s). Also lower startup delay is one of the other main concerns.

\section{$\underline{1.3 \text { Contributions }}$}

This work proposes a design for Video on Demand systems based on distributed streaming of fragmented video. Key contribution of this work is providing a robust Video on Demand architecture that overcomes path congestion coupled with efficient bandwidth sharing for low access latency. Distributed Streaming for Video on Demand successfully extended DVSM to tune in with the Asynchronous Video on Demand mechanism. New algorithms for deciding the streaming sequence of segments are designed. A mathematical relation between the streaming time of a segment and its playback duration is proposed to ensure continuous playback even in case of path congestion. 
The theoretical model is supported with a simulation study conducted in OPNET Modeler to show the effectiveness of the suggested system.

\section{$\underline{1.4 \text { Thesis Organization }}$}

The remainder of the thesis is organized as follows. Chapter 2 gives an elaboration of the background for this thesis which includes DVSM and Asynchronous VoD techniques. Chapter 3 states a formal problem statement for the research. It also states the issues to be considered while devising a solution. Chapter 4 presents Distributed Streaming for Video on Demand with a detailed explanation of its architecture and implementation. Chapter 5 and 6 cover the simulation study and the results obtained. Chapter 7 concludes this research along with possible future work. 


\section{CHAPTER 2}

\section{BACKGROUND}

\subsection{Distributed Video Streaming using Multicast}

Video streaming protocols use multicast for efficient utilization of available network bandwidth. Distributed Video Streaming using Multicast employs multiple servers to stream video streams to a group of receivers. It provides path diversity [8], [11] via multiple servers and provides efficient bandwidth utilization via multicast streaming.

\subsubsection{Introduction}

DVSM uses multicast to stream a video from a server to multiple receivers. Each receiver has a connection to multiple senders such that for each sender there is a multicast group giving rise to multiple multicast groups in the system. A separate reliable channel from the receiver to sender is used for sending control packets. An example with three servers and three receivers is shown in Figure 2.1.

DVSM comprises three major algorithms, bandwidth estimation, rate allocation and packet partitioning. Receivers send out control packets initially that might lead to changes in sending rates and sending sequences at all servers. Rate allocation and a part of bandwidth estimation [10] along with packet partitioning are done at the sender side.

Following is a brief description of the working of DVSM. Initially all the servers start streaming at an equal rate. At some point if one of the paths from sender to 
a receiver gets congested above a predefined threshold, the receiver sends out control packets notifying all the servers about the change in network status. Each server then estimates a new optimal bandwidth and adjusts its sending rate and packets to be sent accordingly. Since the same algorithm runs on all the servers it is guaranteed that each server streams a different sequence of packets to the receivers.

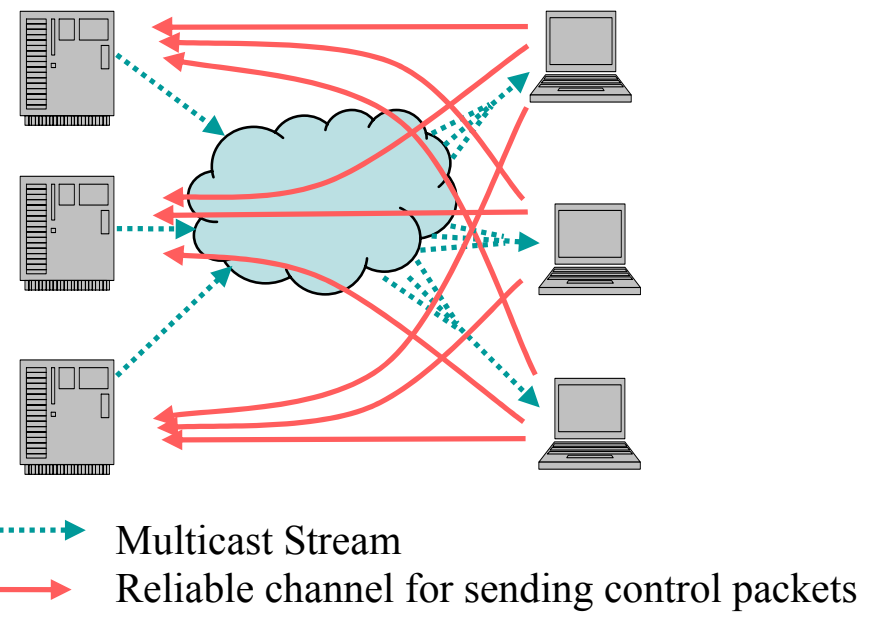

Figure 2.1 A Distributed Video Streaming with Multicast Example

\subsubsection{Bandwidth Estimation}

Bandwidth estimation is done at a receiver to find out the available bandwidth that exists for a path from a sender to a receiver. The formula used to estimate the bandwidth $(B)$ operates on the same lines as in DVS, that is, we use the TCP friendly sending rate estimation formula,

$$
B=\frac{s}{R \sqrt{\frac{2 p}{3}+t_{R T O}\left(3 \sqrt{\frac{3 p}{8}}\right) p\left(1+32 p^{2}\right)}}
$$

$t_{T R O}$ is the TCP timeout, $R$ is the estimated round trip time in seconds, $p$ is the estimated loss rate and $s$ is the TCP segment size in bytes. A receiver estimates bandwidth 
corresponding to each server that it receives video segments from. It then sends control packets to these servers consisting of a sequence of tuples each containing the Delay $\left(D_{j}\right)$, loss rate $\left(L_{j}\right)$ and estimated bandwidth $\left(B_{j}\right)$ for the sender $j$. The last entry, Sync $(S)$ represents the synchronization sequence number used in packet partitioning. At the server information from each control packet received is put into the matrix $S$ at the designated row based on the receiver it was sent from. Row $i$ stores the control packet from Receiver $i$, which contains the Loss, Delay and Bandwidth triples $\left(L_{i j}, D_{i j} B_{i j}\right)$ for each sender $j$ that the receiver is receiving from. We compute the minimum available bandwidth vector $B$ by taking the minimum of a column representing available bandwidth $\left(B_{i j}\right)$ for a sender $j$ as reported by each receiver $i$. This vector is used in deriving the new sending-rates that the senders have to use.

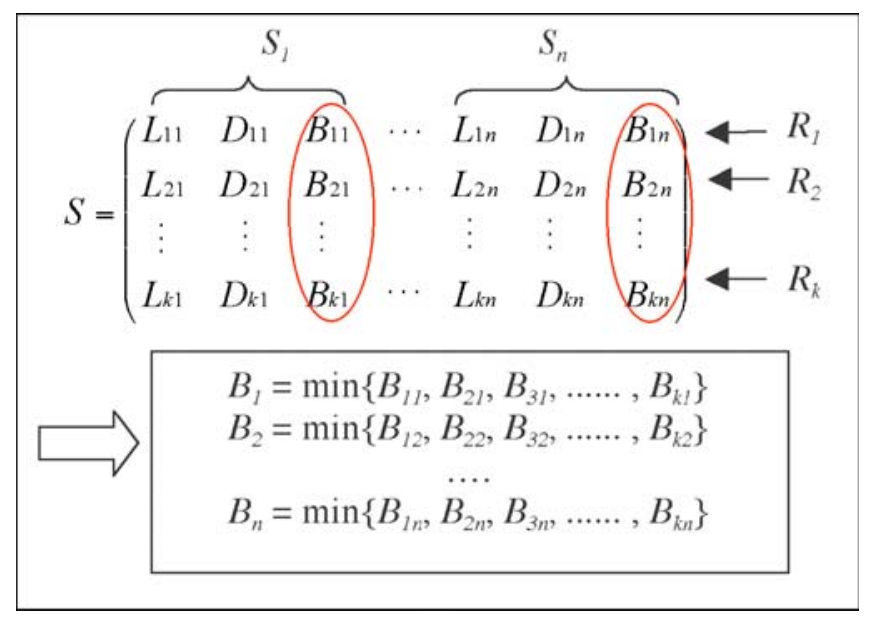

Figure 2.2 Bandwidth Estimation for each Sender

Taking the minimum enables the servers to eventually come to the same bandwidth estimates even though the control packets might arrive in a different order at different servers. 


\subsubsection{Rate Allocation}

Rate allocation in DVSM tries to spread the sending rate evenly among the senders. The idea is to compensate the sending rate of a congested sender by leveraging the rates of less congested sender(s). If a sender's available bandwidth falls below the equally divided sending rate, increasing the rates of other servers compensates the loss in bandwidth of the server. The compensation works by first choosing the server with the lowest loss rate and raising its sending rate to equal its available bandwidth. If this sender's available bandwidth isn't enough for fulfilling the loss, this process continues with next sender having the lowest loss rate until the reduced bandwidth is fully compensated.

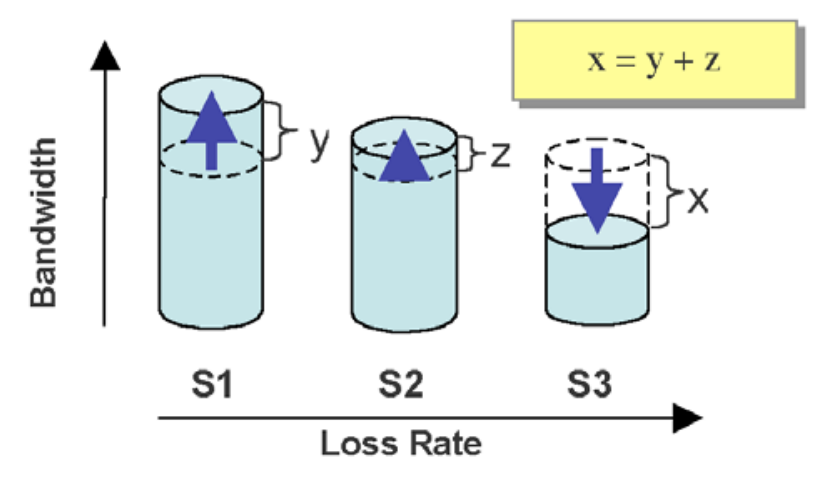

Figure 2.3 Rate Allocation Example

Figure 2.3 shows 3 senders $S 1 \sim S 3$. At the start of the video multicast, all senders are allocated equal bandwidth. Later, $S 3$ 's available bandwidth decreases by $x$ due to network congestion and results in a lower sending rate. To compensate for the lost bandwidth, sending rate at $S 1$ (lowest loss rate sender) is increased until the lost bandwidth is fully compensated or its full available bandwidth (obtained from 
computations discussed in the previous section) is reached (y). As the available bandwidth of S1 alone is insufficient (in this case), S2' sending-rate is increased (by z).

\subsubsection{Packet Partitioning and Synchronization Sequence Number}

The reference point to run the Packet Partitioning algorithm (PPA) is given by the synchronization sequence number (SSN) included in the control packet. Packet partitioning is done to decide which packets are sent from which server. We cannot simply select the SSN in the last received control packet since it might lead to inconsistent packet partitioning resulting in duplicate packets.

The SSN in a control packet is an estimate of sequence number the receiver expects the sender to send. Rather than for retransmission of missing packets it is used as a reference point for doing packet partitioning. Choosing the maximum value of SSN guarantees that senders will arrive at the same SSN. However, there is a very little possibility of missing packets which is tolerable for Video. Figure 2.4 illustrates the working of the effective SSN computation.

\begin{tabular}{|c|c|c|c|c|c|}
\hline 190 & $\begin{array}{lll}30 & \cdots & 80\end{array}$ & \begin{tabular}{|l|}
245 \\
\end{tabular} & $\left.\begin{array}{lll}30 & \cdots & 80\end{array}\right)$ & 250 & $\begin{array}{llll}\ldots & 40 & \cdots\end{array}$ \\
\hline \multirow[t]{4}{*}{$S 1=$} & $\begin{array}{llll}\ldots & 50 & \ldots & 95\end{array}$ & \multirow[t]{4}{*}{$\Rightarrow$} & $\begin{array}{llll}\cdots & 50 & \cdots & 95\end{array}$ & \multirow{4}{*}{$\Rightarrow S 1=$} & $\begin{array}{|llll|}\cdots & 50 & \cdots & 95\end{array}$ \\
\hline & $\left(\begin{array}{llll}\cdots & 25 & \cdots & 90\end{array}\right)$ & & $\begin{array}{llll}\cdots & 35 & \cdots & 90\end{array}$ & & $\begin{array}{llll}\cdots & 35 & \cdots & 90\end{array}$ \\
\hline & $B s 1=25, B s_{2}=80$ & & $B s 1=30, B s 2=80$ & & $B_{s 1}=35, B_{s 2}=80$ \\
\hline & $S_{S 1}=25, S_{S 2}=75$ & & $S_{s 1}=30, S_{s 2}=70$ & & $S_{s 1}=35, S_{s 2}=65$ \\
\hline 9 & $\begin{array}{lll}30 & \cdots & 80\end{array}$ & 250 & $\begin{array}{llll}\ldots & 40 & \ldots & 80\end{array}$ & 245 & $80)$ \\
\hline \multirow[t]{4}{*}{$S 2=$} & $\begin{array}{llll}\text {.. } & 50 & \ldots & 95\end{array}$ & $\Rightarrow S 2=$ & $\cdots \quad 50 \quad \cdots \quad 95$ & $\Longrightarrow S 2=$ & $=\left|\begin{array}{llll}\cdots & 50 & \cdots & 95\end{array}\right|$ \\
\hline & $\left(\begin{array}{llll}\cdots & 25 & \cdots & 90\end{array}\right)$ & & $\left(\begin{array}{llll}\cdots & 25 & \cdots\end{array}\right.$ & & \begin{tabular}{llll|}
$\cdots$ & 35 & $\cdots$ & 90 \\
\end{tabular} \\
\hline & $B_{s 1}=25, B_{s 2}=80$ & & $B s 1=25, B s 2=80$ & & $B S 1=3$ \\
\hline & $S_{s 1}=25, S_{s 2}=75$ & & $S_{s 1}=25, S_{s 2}=75$ & & $S_{s 1}=35, S_{s 2}=65$ \\
\hline
\end{tabular}

Figure 2.4 Example of receiving SSN in differing order

At S1, when a control packet with SSN value of 245 is received it is found to be higher than the current one in use (190), it is therefore selected as a new 
synchronization sequence number for doing packet partitioning. The second control packet received by S1 has SSN value of 250. This is still greater than current value of 245 , so a new value is chosen and packet partitioning is done based on 250. For S2, when a control packet containing a lower SSN is received 250 is retained as the effective SSN. However the sending rates among the senders has changed, so packet partitioning algorithm has to be run based on sequence number 250 .

Table 2.1 Notations for PPA

\begin{tabular}{|l|l|}
\hline$k^{\prime}$ & Sequence number Sync in the control packet which all senders use to initialize the PPA \\
\hline$T_{k^{\prime}}$ & Time at which control packet with sequence $\mathrm{k}^{\prime}$ is sent at the receiver \\
\hline$N$ & Number of senders \\
\hline$P$ & Packet size \\
\hline$T_{k^{\prime}}(k)$ & Playback time for $\mathrm{k}^{\text {th }}$ packet with respect to $T_{k^{\prime}}$ \\
\hline$\sigma(j)$ & Sending interval between packets for sender $\mathrm{j}$ \\
\hline$n_{j, k}$ & Number of packets already sent by sender $\mathrm{j}$ since packet $\mathrm{k}^{\prime}$, and up to packet $\mathrm{k}$ \\
\hline$D(j)$ & Estimated delay from the sender $\mathrm{j}$ to the receiver \\
\hline$S(j)$ & Sending rate for sender $\mathrm{j}$ \\
\hline
\end{tabular}

The main objective of PPA is to ensure that the received packets arrive in an interleaved fashion from multiple senders, so as to reduce the startup delay. After receiving the control packet from the receiver, each sender immediately decides the next packet in the video stream to be sent using the PPA. All the senders simultaneously run this algorithm in a distributed fashion in order to ensure that, all packets are sent by 
one and only one sender, and also to minimize the startup delay. To explain the packet partitioning algorithm we use the notation in table 2.1.

If the reference time, $T_{k}$, is conceptually chosen to be the departure time of the control packet from the receiver, the estimated arrival time of the $k^{\text {th }}$ packet sent by sender $j$ is $n_{j, k} \sigma(j)+2 D(j)$. This is because it takes $D(j)$ for the control packet to arrive at the sender $j, n_{j, k} \sigma(j)$ for the $k^{t h}$ packet to be sent by sender $j$, and $D(j)$ for it to arrive at the receiver.

Since $T_{k},(k)$ is also the playback time of the $k^{\text {th }}$ packet with respect to $T_{k}$, the expression $A_{k^{\prime}}(j, k)=T_{k}(k)-\left[n_{j, k} \sigma(j)+2 D(j)\right]$ can be interpreted as the estimated time difference between arrival and playback time of the $k^{\text {th }}$ packet, if sender $j$ is its originator. If $A_{k^{\prime}}(j, k)$ is positive, $k^{\text {th }}$ packet is on time, otherwise, $k^{\text {th }}$ packet is late. Hence, maximizing $A_{k}(j, k)$ is equivalent to minimizing the probability that the $k^{\text {th }}$ packet is late.

The basic idea is that among all senders $j=1, \ldots N$, the one that maximizes $A_{k^{\prime}}(j, k)$ is assigned to send $k^{\text {th }}$ packet. Specifically, each sender computes $A_{k^{\prime}}(j, k)$ for each packet $k$ for itself and all other senders, and only sends $k^{\text {th }}$ packet if it discovers that $A_{k^{\prime}}(j, k)$ is at a maximum for itself. If $A_{k^{\prime}}(j, k)$ is not at a maximum for sender $i$, it will increase $k$ by one, and repeats the procedure until it finds the packet $k^{*}$ for which $A_{k},(j, k)$ is at a maximum among all other senders.

Each sender effectively keeps track of all the values of $A_{k^{\prime}}(j, k)$ for all $N$ senders and updates $A_{k}(j, k)$ every time a packet is sent. The values of $A_{k^{\prime}}(j, k)$ evolve in the same way at all senders even though they are computed at different locations. The reasons for 
this are that all the senders (1) receive the same control packet from the receiver, (2) only use the information in the control packet to update $A_{k^{\prime}}(j, k)$ and (3) all use the same equation to do so. Therefore there is neither any need for information exchange among the senders nor a synchronized clock at various senders.

All the needed information to compute $A_{k}(j, k)$ is in the control packet. The estimated delays $D(j)$ are explicitly in the fields D1-D5 of the control packet. Secondly, $\sigma(j)=P / S(j)$ is easily computed from fields S1-S5 in the control packet. Since $T_{k}(k)$ does not affect the value of $j$ that maximizes $A_{k},(j, k)$, for convenience, it can arbitrarily be set to any value, say zero, by all users for all packets $k$. As for $n_{j, k}$, we have $n_{j, k}=0$, for computing $A_{k^{\prime}}(j, k)$ where $k^{\prime}$ is the synchronization sequence number as specified in the sync field of the control packet. Therefore for packet $k$, all senders can determine the sender $j^{*}$ with the largest $A_{k^{\prime}}(j, k)$ chosen to send packet $k^{\prime}$. Each time sender $j$ is chosen to send a packet, $n_{j, k}$, is incremented by one at all sender locations. Since all senders are initialized to start computing $A_{k^{\prime}}(j, k)$ for packet $k^{\prime}$ onwards, and they all receive identical information as supplied in the control packet to update $A_{k} \cdot(j, k)$, the values of $A_{k^{\prime}}(j, k)$ at all the senders evolve the same way, and as such the decision as to which sender maximizes $A_{k},(j, k)$ for $k>k^{\prime}$ is identical at all senders, and hence no duplicate packets are sent.

\subsubsection{Summary}

DVSM is a multicast protocol for video streaming which provides path diversity via distributed server setup. It efficiently uses multicast for economic utilization network bandwidth. The algorithms proposed adjust sending rates as well which server 
sends next according to network congestion based on receiver feedback. This work covers the bandwidth allocation, rate allocation and packet partitioning algorithms that comprise DVSM.

\subsection{A New Asynchronous Hybrid Mechanism for Video on Demand}

This work introduces a new algorithm that combines the best features of Broadcasting and Patching [12] [13].

\subsubsection{Introduction}

In Video on Demand systems the goal is to maximize the use of broadcasting to effectively share network bandwidth such that patching is only required at the minimum to make up for the lack of time-dependence between user requests. The proposed algorithm is based on segmentation-based Broadcasting and Patching. There is no constraint of synchronicity on the video streams which reduces the complexity of the system. Since the video are segmented the system can provide VCR like functionality very efficiently.

\subsubsection{Assumptions}

Before we proceed to the algorithm let us get familiar with the terms used and the assumptions made in the analytical model. Table 2.2 gives a list of notations used in the description of the model.

They make an assumption that there is support to establish multiple channels for both group multicast and point-to-point unicast. Also the channels can operate at differing rates through a simple bandwidth partitioning mechanism like weighted fair queuing. The video is perceived in units of time rather than data-length. A video of 
length $L$ seconds is fragmented into equal size segments ( $K$ of them) of length $L_{s}$ seconds each. $L_{s}$ is given by $L / K$. The actual segment length (in bits) is $(L / K) \times b$.

Table 2.2 Terminology used in the VoD Model

\begin{tabular}{|l|l|l|}
\hline Parameter & Symbol & Value(s) \\
\hline Total bandwidth of server & $B$ & $150,300 \mathrm{Mbps}$ \\
\hline Number of videos & $M$ & $10-20$ \\
\hline Length of each video & $L$ & $120 \mathrm{x} 60$ seconds \\
\hline Display/coding rate of video & $b$ & $1.5 \mathrm{Mbps}$ \\
\hline Ratio of Multicast to Patching b/w & $\alpha$ & $0.0-1.0$ \\
\hline Bandwidth for multicast & $B_{m}$ & $\alpha \mathrm{x} B$ \\
\hline Bandwidth for Patching & $B_{p}$ & $(1-\alpha) \mathrm{x} B$ \\
\hline Number of segments per video & $K$ & $<$ derived $>$ \\
\hline Length of each segment (in secs) & $L_{s}$ & $L / K$ \\
\hline Number of multicast channels & $m$ & $<$ derived $>$ \\
\hline Number of patching channels & $p$ & $<$ derived $>$ \\
\hline Arrival rate of requests (per sec) & $\lambda$ & - \\
\hline
\end{tabular}

\subsubsection{Algorithm}

The goal is to maximize the use of broadcasting to effectively share network bandwidth, and using patching to address the issue of lack of time-dependence between user requests. In a nutshell, if a request comes when the broadcast of a video has already begun, then we patch the part that the request has missed in such a manner as to ensure that the request is able to catch up soon with the broadcast. Using segmented video allows limiting the amount of patching to a maximum of one segment and periodic 
broadcasting helps in serving the request through broadcast at least from the second segment onwards. Following are some preliminaries before we proceed to the algorithm.

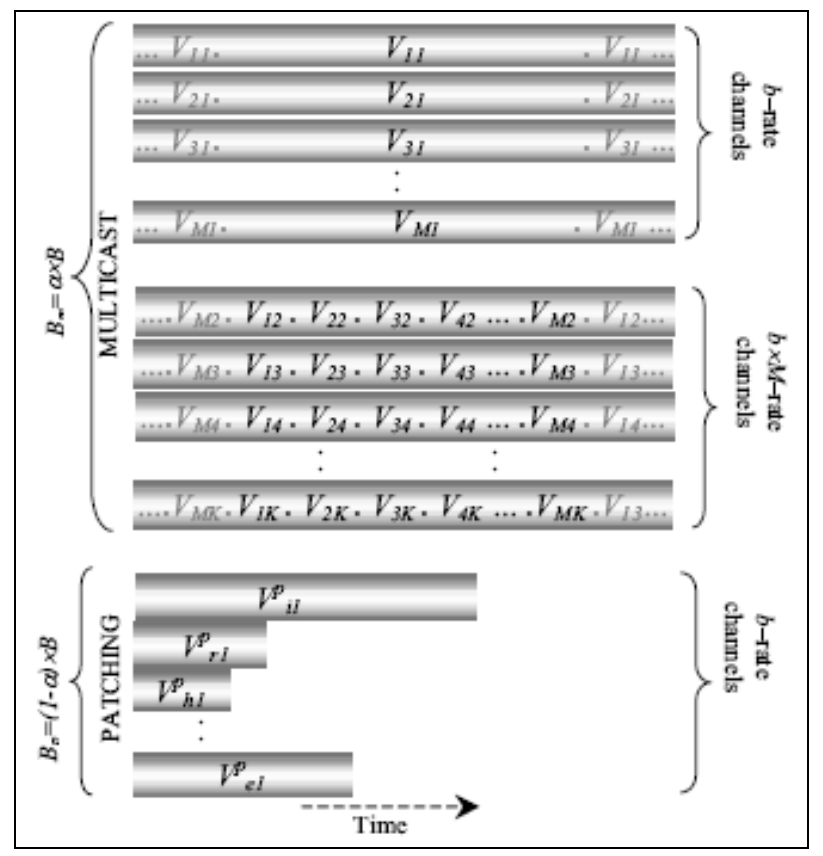

Figure 2.5 Channels: Partitioning and Bandwidth

The total bandwidth $B$ is divided in the ratio of $[\alpha: 1-\alpha]$ among the multicast $(m)$ and patching $(p)$ channels. The multicast channels are divided into two categories as shown in Figure 2.5.

- The first category $(m l)$ contains $M$ channels each of bandwidth $b$, which are each used to broadcast the first segment, $V_{X I}$ of the $X^{\text {th }}$ popular video. The segment is transmitted continuously in cyclic manner. The $i t h$ channel is denoted $C^{m l} i$.

- The second category $(m 2)$ contains $K-1$ channels each of bandwidth $b x M$, which are used to broadcast segments as follows: The ith channel (denoted $C^{m 2}{ }_{i}$ ) broadcasts the $(i+1)$ th segment of each of the $M$ videos in turn cyclically. For example, the first 
$m 2$ channel $\left(C^{m 2}{ }_{1}\right)$ broadcasts $V_{12}$ followed by $V_{22}$ followed by $V_{32}$ and so on till $V_{M 2}$. This sequence is repeated cyclically.

The $p$ patching channels are unicast channels, each transmitting at the rate $b$. The content of these channels depends on what is being patched, denoted by $V^{p} y l$ to indicate that a part of the first segment of the $y^{\text {th }}$ video is being transmitted. The patching channel is always used to only patch a part (the missed part) of the first segment of a requested video.

We now discuss the working of the algorithm. The first segment of each video is being broadcast continuously in a cycle on a dedicated channel. If a client request arrives in the middle of the first segment's transmission, it may have to wait for the segment to complete transmission before starting reception in the next cycle. Patching is used to prevent this possibly long delay. Depending on when a client request arrives, its expected wait time $\left(w^{e}\right)$ varies and accordingly, its handling:

- Case A: If a client request comes within $\delta$ (threshold) seconds of the end of the current broadcast of the first segment, then we force the request to wait till the start of the next cycle of broadcast. We express $\delta$ in terms of $L_{s}$, the time-length of the segment. Accordingly, $\delta$ is given values $L_{s} / 8, L_{s} / 6, L_{s} / 4, L_{s} / 3$ etc.

- Case B: If a client request comes at any other time, then we patch the part from the beginning that the request has missed, while requiring the client to buffer the current broadcast. Therefore, the client will receive and display the patched video, while simultaneously buffering the rest of the segment from the ongoing broadcast. 
In the second case, once the patch is played, the client continues by playing the buffered broadcast and once this finishes, it can play the second segment from the corresponding broadcast. Just as the client was buffering the remaining part of the first segment while receiving a patch, it could also buffer the second segment from a corresponding broadcast. The first segment will play continuously by getting content, first from the patch and then from the buffered part of the first segment. However, in order to ensure continuity of play beyond the first segment, the client must be able to start buffering the second segment before the first segment completes play. In order to meet this requirement for the consecutive segments also the following condition must be satisfied:

$$
L_{s} \geq \frac{L_{s} \times b}{B_{m} / K} \times M
$$

In the worst-case it would take one complete cycle (of a $m 2$ channel) to start the transmission of the next segment, which gives us the right hand side of the equation. Simplified form of this relationship gives us the value for $K$,

$$
K \leq \frac{B_{m}}{b \times M}
$$

\subsubsection{Access Latency for a User Request}

The aim is to reduce the waiting time for a user request in the VoD system. A client request faces two possible cases, Case $A$ occurs with probability $P_{A}=\delta / L_{s}$, and Case $B$ occurs with probability $P_{B}=\left(1-P_{A}\right)$. The average waiting time in Case $A$ is $\delta / 2$, since a request that falls within $\delta$ time units before the end of the current broadcast will 
have its wait time uniformly distributed between 0 and $\delta$. Therefore the waiting time in case $A$ is given by:

$$
W_{A}=\frac{\delta}{L_{s}} \times \frac{\delta}{2}
$$

The patching service is modeled by an $\mathrm{M} / \mathrm{U} / \mathrm{q}$ queuing model where the request arrival distribution is Poisson (the inter-arrival times are exponential) and the service time follows a Uniform distribution, with $q$ servers available for service. Assuming that the arrivals to $\mathrm{VoD}$ system are Poisson the arrivals to Case $B$ after accounting for Case $A$ are also Poisson. $\lambda$ being the arrival rate to the $\mathrm{VoD}$ system, the arrival rate for Case $B$ is given by:

$$
\lambda_{B}=\left(1-\frac{\delta}{L_{s}}\right) \times \lambda
$$

The patching service times are uniformly distributed between 0 and $(L s-\delta)$ since only those requests that fall beyond the $\delta$ threshold require patching. The maximum patching amount therefore is, $(L s-\delta)$ and the minimum 0 . Thus average service time:

$$
T_{s}=\frac{\left(L_{s}-\delta\right)}{2}
$$

The last parameter for the queuing model, the number of servers, $q$ is observed to equal the number of patching channels, $(p+1)$. If a request waiting for patching service is delayed for too long then it may be possible to serve it through the next broadcast from the channel with respect to which the patching was initiated. That is, we can perceive the next broadcast as a patching channel for this request, resulting in $p+1$ 
servers. As the model is not a standard $\mathrm{M} / \mathrm{M} / \mathrm{m}$ model, we use the approximation for waiting times in a $\mathrm{G} / \mathrm{G} / \mathrm{m}$ model.

$$
W_{B} \approx \frac{E_{c}(q, u)}{q(1-\rho)}\left(\frac{C_{v}^{2}+C_{S}^{2}}{2}\right) \times T_{s}
$$

where $C^{2}{ }_{v}=1$ is the coefficient of variation for a Poisson process and $C^{2}{ }_{s}=1 / 3$ is the coefficient of variation for a uniform distribution; $u$ is the traffic intensity given by $\lambda_{B} \times T_{s} ; \rho$ is the server utilization given by $u / q$; and lastly $E_{c}(q, u)$ is the Erlang-C function. [15]

The waiting time that a request may suffer is given by the two cases $(A$ and $B)$ as discussed above. Accordingly, the waiting time is:

$$
P_{A} \times W_{A}+P_{B} \times W_{B}
$$

\subsubsection{Summary}

This work presents a combination of two popular video on demand techniques to give an elegant approach to VoD systems. It covers an analytical model of the user access latency in a generic $\mathrm{VoD}$ system. Along with a performance upgrade the system is able to provide VCR like functionality with less complexity. 


\section{CHAPTER 3}

\section{PROBLEM STATEMENT}

Video streaming on the Internet has to take account of the unreliable, best effort service of the network. Distributed video streaming using multicast utilizes path diversity of multiple senders to provide a more reliable or timely way of streaming as well as efficient utilization of bandwidth. But DVSM has been implemented for a one time telecast and neither can receivers join the telecast in an ad hoc manner. We want to extend the DVSM technique to provide true Video-on-Demand service. In short the system designed should enable users to join the streaming at any point and still catch the entire video with VCR like functionality provided.

As per VOD framework different clients may or may not be watching the same video, for example different movies. Also clients watching the same movie may not be watching the same video clip. Each client receives video streams from at least two servers enabling path diversity in case of congestion. There are a number of issues to be considered here. Where do the important decisions of sender assignment to a particular receiver, which parts of the video are streamed by that sender, etc. decisions take place? We need to find out the modifications if any to the bandwidth estimation, rate allocation, and packet partitioning algorithms. We also need to determine the content distribution; which videos are stored on each sender. Video popularity is an essential parameter by which we can save storage without reducing performance. [9] The 
distribution of video segments to be multicast from different senders should be done such that there is continuity in the video playback i.e. the before the previous segment is received and played completely the reception of the next segment should start.

The feedback mechanism in DVSM was completely distributed and every sender determined what to send next and when to send at what rate. We need to determine here if the feedback is to go via a central authority or be completely distributed as in DVSM. We shall look at all these issues and their solutions in detail in the following chapters. 


\section{CHAPTER 4}

\section{DISTRIBUTED STREAMING FOR VIDEO ON DEMAND}

In the previous chapters we have seen two techniques to implement video streaming viz. DVSM and asynchronous Video on Demand. The salient features of these two techniques are combined to design a distributed asynchronous VoD system in this work.

\section{$\underline{4.1 \text { System Model }}$}

The entire working in DVSM is distributed in the sense the decision making is not confined to any particular server, but each server runs the designed algorithms independent of others. It is assured that the servers arrive at the same conclusions by running the same algorithms on the same control packet values at all the servers.

To design a true Video on Demand system where multiple users request different videos at different times we need to incorporate the asynchronous VoD technique in our distributed design. Thus we use a central server to perform the initial multicast group management tasks to allow users to join the system in an ad-hoc manner. We also use segmented videos which allows us to limit the amount of patching to one segment as in the Asynchronous $\mathrm{VoD}$ technique. Also periodic multicasting helps to serve the request through multicast at least from the second segment onwards. 


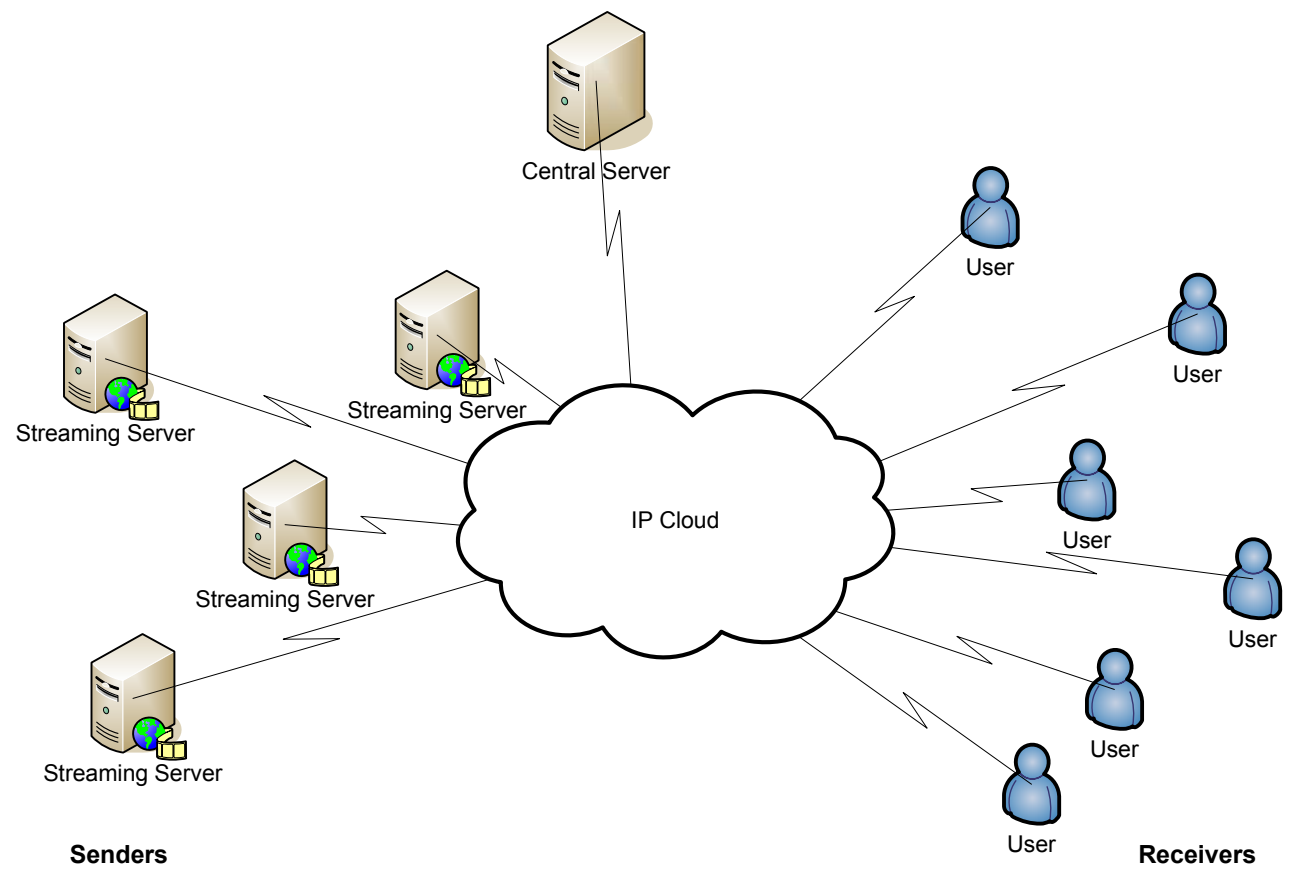

Figure 4.1 System Model

The system comprises a Central Server, subordinate servers, referred to as senders in the remainder of the document and receivers or clients. The senders store all the video content of $\mathrm{M}$ popular videos. The central server controls the distribution of requests from receivers to the senders. It also takes care of streaming the first video segment as well as patching the part of the first segment to receivers that have missed it. This helps in reducing the wait time for each request since it is patched the first segment while its request is being forwarded to one or more secondary servers which will stream the remaining video. Thus a receiver joins the multicast of a number of senders to receive different segments of the video from different servers. Also client requests for videos that do not fall into one of these popular videos are handled by patching the entire video over a unicast channel from the Central Server. 
A video of length $L$ is divided into segments $(K)$ of equal size which are sequentially streamed from different senders. As part of the system initialization the Central Server schedules the multicast of the first segment of all the videos cyclically over $M$ channels each of bandwidth $b$. Segment $V_{X I}$ is the first segment of the $\mathrm{X}^{\text {th }}$ popular video. Figure 4.2 shows the channel partitioning on the Central Server.

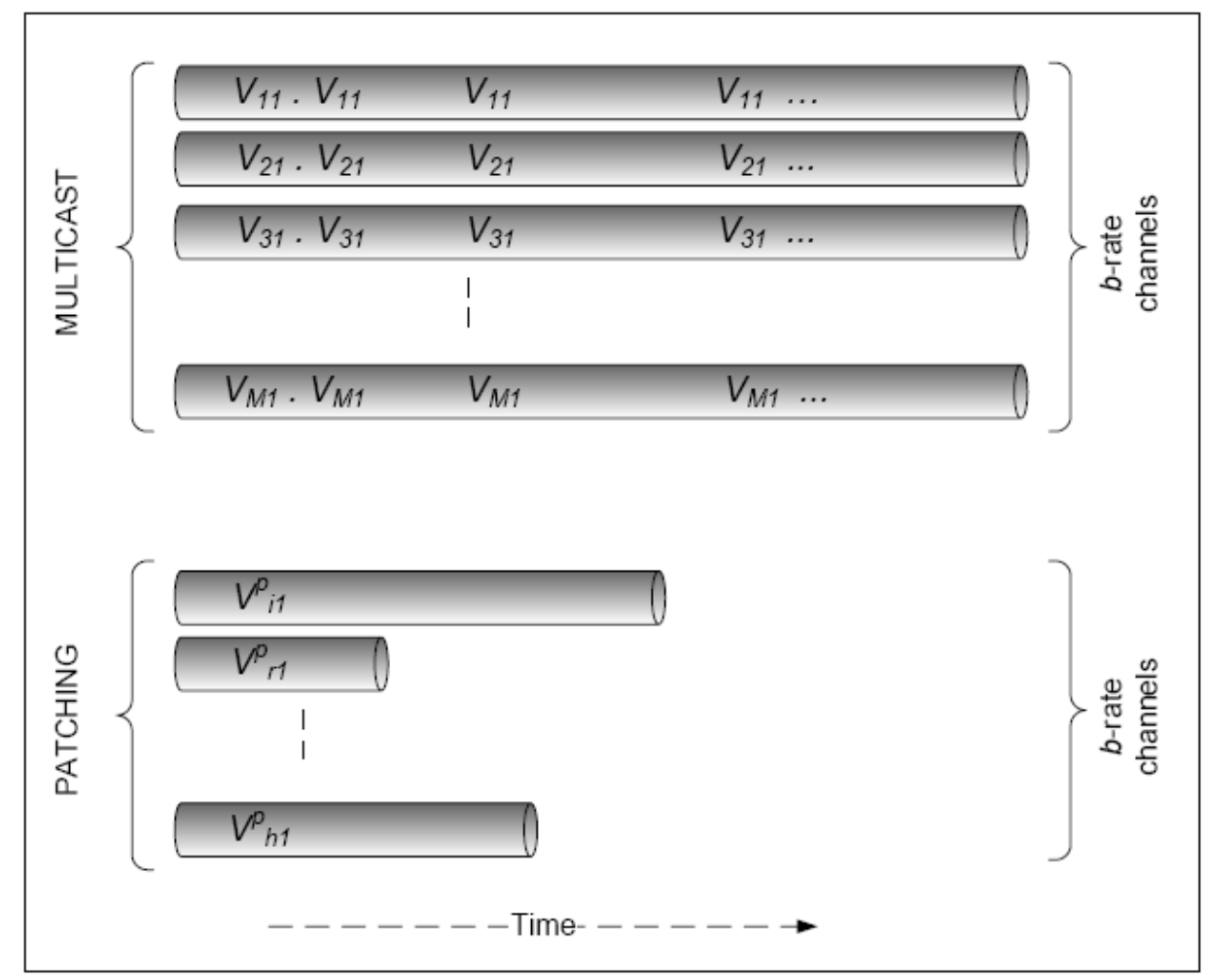

Figure 4.2 Channel-Partitioning on Central Server

The Senders simultaneously schedule the multicast of the remaining $K-1$ segments of all the videos on $K-1$ channels each of bandwidth $2 x b x M$. The $i^{\text {th }}$ channel schedules the $(i+1)^{t h}$ segments of all the $M$ videos in a cyclic manner. Out of the $M$ video segments that are scheduled only those which are being requested and assigned to this sender by the Central Server are actually multicast. The significance of the different 
rates of the channels is that a video segment would have different lengths in terms of transmission time on the $b$-rate and the $2 \times b x M$ rate channels.

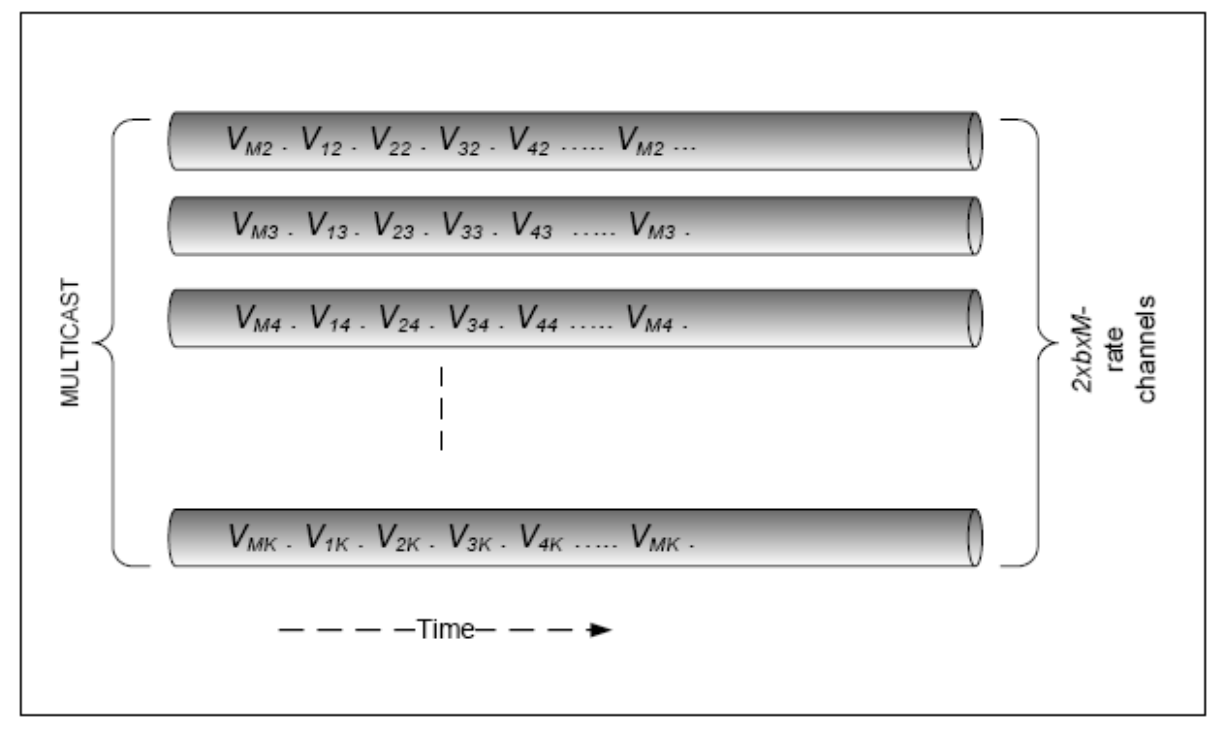

Figure 4.3 Channel-Partitioning on Senders

At time $t$, a client sends a video request to the Central Server. The central server (CS) calculates its estimated wait time $\left(w^{e}\right)$ and accordingly responds:

Case $A$ : If the request falls within $\delta$ seconds of the end of the current multicast of the segment then the request is made to wait till the start of the next cycle of multicast of the first segment of the requested video. Also the Central Server sets up $N$ senders that will start streaming the consecutive segments with minimum delay and notifies the receiver to join the multicast of these $N$ senders. For e.g. for $N=3$, the Central Server selects 3 servers to multicast the second, third and fourth segment respectively. So the receiver joins $N$ multicasts for $N$ segments, one for each.

Case B: If a request comes at any other time, the Central Server patches the part of the segment that the request has missed. Also the Central Server seeks $N$ senders 
that will start streaming the consecutive segments with minimum delay and notifies the receiver to join the multicast of these senders.

Consequent requests are handled in the same manner with each receiver joining the multicast of $N$ senders. Initially the servers are assigned the transmission of one segment each and are scheduled to transmit the segments in a cyclic order. In our example, first sender streams segment 2, second streams segment 3, third streams segment 4 and then the first server also sends segment 5 (on a different channel than that for segment 2) and so on.

If at some point in time one of the paths from a sender to a receiver experiences congestion above a predefined status resulting in packet loss and delay, the receiver sends out control packets to all the senders who are sending multicast streams to it. When a control packet is received at a sender it runs the segment partitioning algorithm. Thus each sender adjusts to the network congestion by streaming a different sequence of segments to its receivers.

\subsection{Multicast Schedule on Senders}

When the Central Server receives a client request it notifies the receiver to join the multicast of $N$ channels where $N$ segments starting from the second segment are scheduled for multicast, one on each after an interval of one segment-playback time. After two segment-playback intervals with respect to the end of the current segment transmission each sender schedules the multicast of a segment which is calculated as currently streamed segment number $+N$. E.g. the $1^{\text {st }}$ server schedules the multicast of the $5^{\text {th }}$, so that when the receiver starts getting the $4^{\text {th }}$ segment it also starts buffering the 
$5^{\text {th }}$ segment, to maintain continuity in playback. Figure 4.4 shows the worst-case time by which each segment is streamed by the respective senders during the initial schedule.

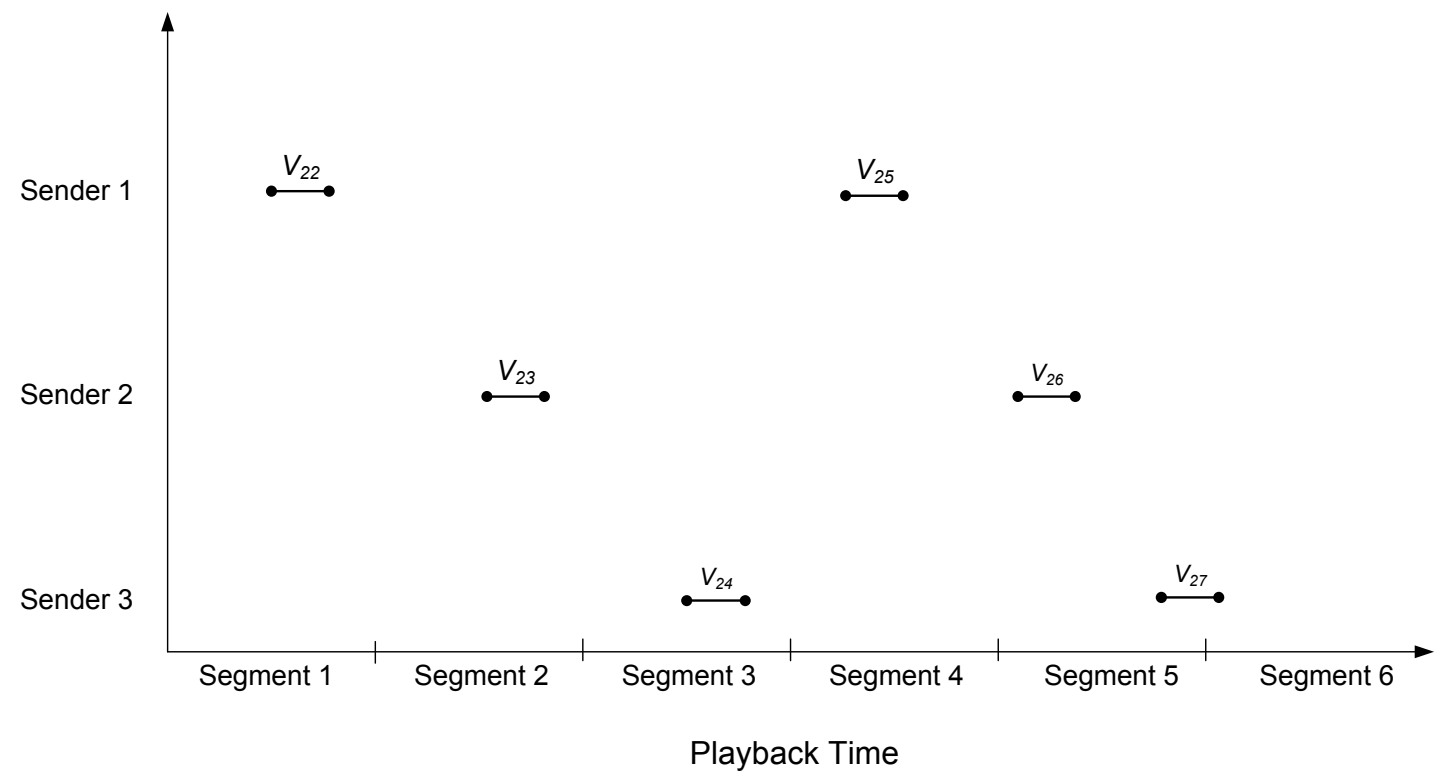

Figure 4.4 Initial Segment Schedule on Senders

When a control packet is received the segment schedule is changed according to the segment partitioning algorithm explained further ahead in this chapter.

\subsection{Bandwidth Estimation \& Sending Rates}

Bandwidth estimation is done to find out the available bandwidth for a path from a sender to a receiver. The receivers calculate the estimated bandwidth for senders using the loss rate and delay for each sender and send control packets accordingly.

Rate allocation in DVSM is needed in order to keep the total sending rate from all the senders steady. This is required in order to maintain the continuous streaming of packets for smooth playback. Distributed Streaming for Video on Demand does not 
require rate allocation [2] for two reasons. Firstly the receivers always start buffering the segment before its playback begins. So it is not required to stream the segment with a higher rate in case of path congestion. Secondly the rates of the channels are set in a way to maintain playback continuity at the receiver. Changing these rates would disrupt the segment schedule. Thus rate adjustment is neither required nor permissible.

\section{$\underline{4.4 \text { Control Packets and Synchronization Sequence Number }}$}

In Distributed Streaming for $\mathrm{VoD}$ there are many senders streaming different segments of different videos to receivers. When a sender receives a control packet it has to identify the receiver that has sent it in order to determine the segment streaming sequence of which video multicast has to be changed. Also since we do not need rate allocation the bandwidth estimates in the packets are not required [2]. Thus the control packet format is modified from that in DVSM to include information about the multicast group, the packet number and the segment number that the receiver wants to request next. The (Delay, loss-rate) values are repeated for number of senders $N$.

\begin{tabular}{|l|l|l|l|l|l|l|l|l|l|}
\hline D1 & L1 & D2 & L2 & $\ldots$ & Dn & Ln & M & Packet & Sync \\
\hline
\end{tabular}

Figure 4.5 Control Packet Format

Sync represents the synchronization sequence number and is the next segment requested by the receiver. It is used for initializing the segment partitioning algorithm. $\mathrm{M}$ stands for the multicast group of the receiver and Packet stands for the estimate of the packet number that the sender might be sending at the time when the control packet arrives. 
In DVSM control packets are sent based on delay in packet reception and there may be many control packets arriving close to each other that may have differing arriving order among the senders. Using the Sync on the last received control packet may result in different Sync chosen among the senders, leading to inconsistent packet partitioning. Hence the Sync is treated as an estimate of the sequence number the sender would be sending at the arrival of the control packet. Thus the maximum value of Sync is chosen.

In Distributed video streaming for $\mathrm{VoD}$ consecutive segments are already scheduled to be sent from different senders. Also the segment length being moderately large we can have control packets sent based on packets received for a segment i.e. at most one control packet per segment. Thus there are very few control packets. Also the segment length is much larger as compared to packets in DVSM and hence the chances of receiving delayed control packets i.e., control packet for segment 3 arrives after control packet for segment 4 , are none.

The receivers are always buffering the segment next to the one which is currently being played, in order to maintain continuity in playback. Thus when it finds packet loss in the reception of a segment it sends a control packet with the segment number field set to that segment number. Again since the segment size is large the complete requested segment cannot be in transit and not yet reached the receiver. Thus the receiver notifies the exact segment number while an estimate of the packet number that has to be sent next by the senders. 


\section{$\underline{4.5 \text { Segment Partitioning Algorithm (SPA) }}$}

When a sender receives a control packet, it immediately runs the SPA to determine the next sequence of segments in the video stream to be sent. All of the senders arrive at the same conclusion since they use the same copy of the control packet to run the same algorithm. The sender that maximizes estimated time difference between the arrival and play back time of each segment is selected to stream the next segment.

The notations used in the algorithm are similar to the Packet partitioning algorithm in DVSM [2] with one exception; $k$ ' which is now the next segment number requested in the control packet which all senders use to initialize the segment partitioning algorithm. The algorithm works in the same manner to determine the sender who sends the next sequence of packets remaining in the segment. The segment count is initialized to the requested segment number in the control packet. Each sender determines the estimated time difference between arrival and playback time of the requested packet number in the control packet. All the senders can determine the sender who maximizes this value and then that sender sends the requested part of the segment. Every time a sender is selected to stream the next segment the segment count is incremented by 1 . After that the segment partitioning algorithm continues by selecting a sender to send the next segment i.e. after every half a segment playback interval each server calculates the value of $A_{k}(j, k)$ and the one that maximizes it sends the next segment. Thus the initially set multicast schedule by the Central Server is changed after control packets are received to adjust to the network status. 


\section{$\underline{4.6 \text { Playback Continuity }}$}

In the case when a part of the first segment is patched, the user continues to play the buffered multicast and then plays the second segment from the corresponding multicast. It could also buffer the second segment just as it was buffering the first segment while receiving the initial patch. Thus in order to maintain continuity in play the user must be able to start buffering the second segment before the first segment completes play. It is required that the receiver can buffer the next segment even in case of congestion where it has to receive the segment from a different sender after receiving a part from the first sender. The following constraint must be satisfied:

$$
\frac{L_{s}}{b} \geq 2 \times \frac{L_{s}}{\left(B_{m}-b \times M\right) /(K-1)} \times M
$$

The time that it takes to playback a segment must be greater than or equal to twice the time that it takes for the server to start transmission of the next segment. This ensures that in one segment playback time the next segment is scheduled for multicast twice. Thus during a segment transmission, if the receiver notifies congestion on the path from one sender the remaining part of the segment is streamed from another sender within the previous segment playback time. This condition gives the value of $K$, the number of segments to fragment the video into.

$$
K \leq \frac{B_{m}-b \times M}{2 \times b \times M}+1
$$




\section{$\underline{4.7 \text { Buffer Space Requirement }}$}

Due to segmentation of the video the buffer space requirement at the client is limited to a value. In the worst case the buffer size is two segments $(2 L s)$. In the worst case the $i^{\text {th }}$ segment is being received and played, the $(i+1)^{\text {th }}$ segment would have to be buffered for continuity. While the $(i+1)^{t h}$ segment is being played from the buffer the $(i+2)^{t h}$ segment has to be buffered, therefore requiring two segments worth of buffer.

\section{$\underline{4.8 \text { Summary }}$}

Distributed Video Streaming for VoD combines two video streaming techniques. This chapter investigates some of the key issues that arise due to ad-hoc arrival of user requests. It ensures compatibility between the two base techniques by suggesting modifications to the algorithms and constraints implemented in each. 


\section{CHAPTER 5}

\section{SIMULATION STUDY}

The model was tested using OPNET Modeler [16] for validity and performance. “OPNET Modeler is the industry's leading environment for network modeling and simulation, allowing one to design and study communication networks, devices, protocols, and applications with unmatched flexibility and scalability". The Modeler has an object oriented modeling framework which simplifies mapping the real system to the designed model. Finite state machine (FSM) modeling was used to simulate the protocol behavior. Process and node models with full functionality for the Central Server, Senders and Receivers were designed and implemented.

\section{$\underline{5.1 \text { Model }}$}

The entities designed for the network are Central Server, senders, receivers, routers, links and TCP senders and receivers. Following is a brief description of their design and functionality. The node models for all the nodes consist of a processor where the actual algorithms are implemented and the receiver and transmitter objects, which determine the number of interfaces the entity has. The process models are event driven FSMs that implement various functions of the node.

- Central Server- The Central Server FSM consists of five states. The 'init' state initializes the state variables and sets the necessary fields for the simulation. The Central Server schedules the multicast of the first segment of all the videos 
using the 'multicast_pk' state. The ' $r c v$ join' state handles user requests by streaming them the first segment, either by patching or multicast and then adds them to the multicast of the senders for consecutive segments. The senders send control packets to inform the Central Server about the segment they would stream next. These packets consist of information about the video number the sender will transmit next on each of its channels. In the 'sender_info' state this info is updated. The 'record_stats' state stores the latency-arrival rate values for each simulation.

- Sender- The sender FSM has four main states to perform four different functions. The ' $x m t$ ' state handles the scheduling and multicast of segments on the respective channels. When a control packet arrives, the 'adjust_sc' state stores the information contained in the packet and runs the segment partitioning algorithm to determine sender that sends the next segment. The 'segment_part' state then runs the same algorithm every time a segment streaming is complete. The 'update_multsc' state receives a control packet from the sender and schedules the requested segment on the corresponding channel.

- Receiver- There are two main states in which the receiver can be; viz 'process_pk' and 'sampling_intv'. In the first state a packet is received from the sender and receiver calculates different values needed for bandwidth estimation. The actual bandwidth estimation algorithm is run periodically after an interval of $100 \mathrm{~ms}$ in the second state. 
- Router- The routers implement basic functions such as routing packets and updating routing tables when a new receiver joins the system.

- Link- The link model represents a network link. It uses the Modeler's propagation and transmission delay models.

- TCP senders and receivers- These are used to model congestion on a path. These are simple nodes generating TCP packets and receiving them as specified by the packet arrival distribution.

\section{$\underline{5.2 \text { Simulation Scenario \& Parameters }}$}

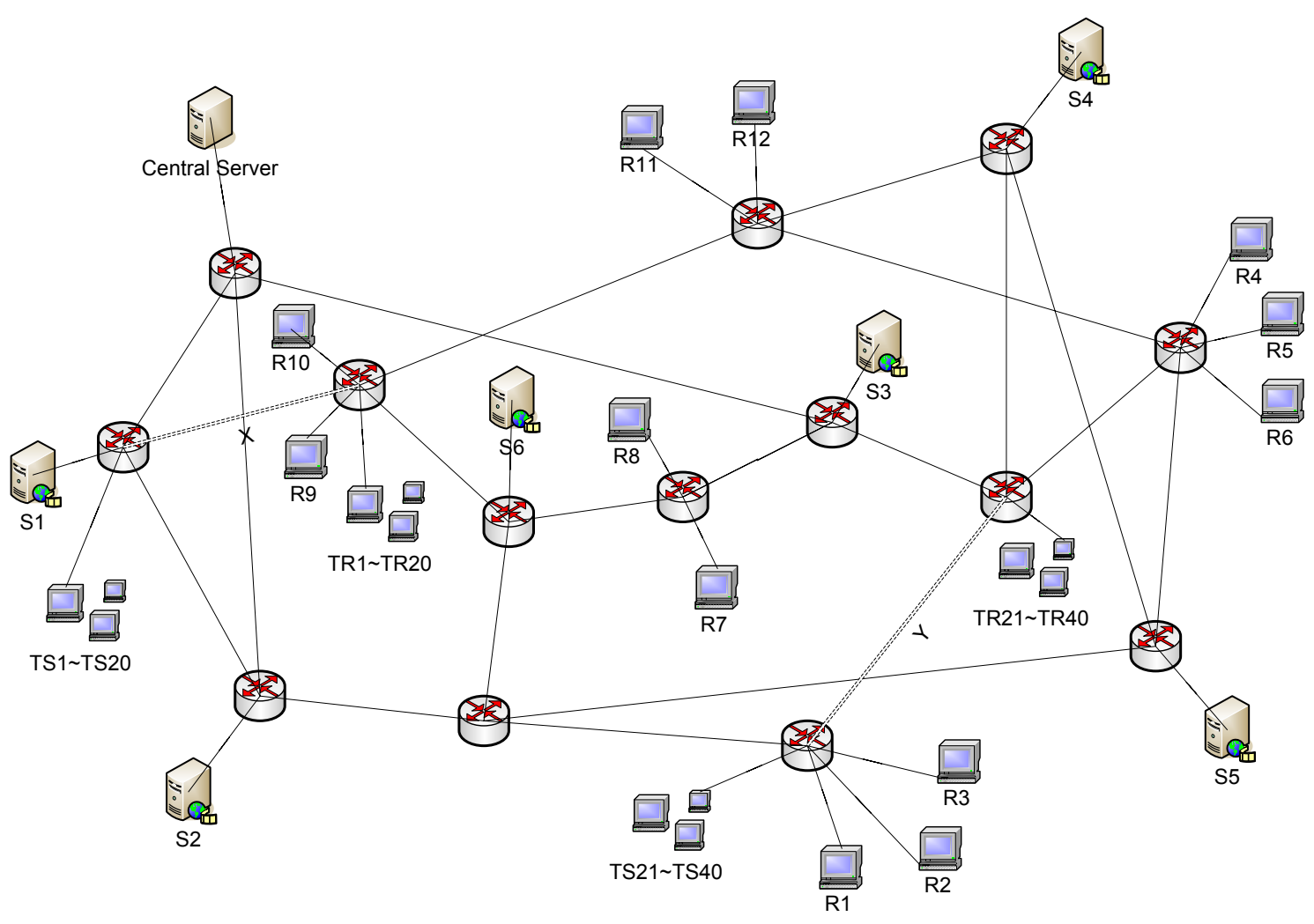

Figure 5.1 NSFNet Backbone Network for Simulation

The simulation was carried out using 3 and 6 senders in turn to see the impact of $N$, the number of distributed servers on throughput and average latency. Figure 5.1 
shows the network model with the positioning of the Central server, senders and receivers. For the first simulation run $\mathrm{S} 1 \sim \mathrm{S} 3$ are active. For the second run $\mathrm{S} 1 \sim \mathrm{S} 6$ are active.

NSFNet backbone network ${ }^{1}$ (July 1989-November 1992) was used for the network topology. In each scenario, there exists congestion links shared with other TCP traffic. All the links are $100 \mathrm{Mbps}$ except the congestion links which are $100 \mathrm{Kbps}$ each. During the simulation period, burst of TCP traffic is started and stopped to test how Distributed Streaming for Video on Demand compares to DVSM [2]. A video of length 120 minutes is streamed to the receiver(s). Each receiver is assumed to have buffering capability of $2 L s$. The buffer is used to store the consecutive segment.

In the first scenario, we elaborate on the performance of our strategy with respect to latency when varying the parameters of interest. We study the impact of bandwidth partitioning, threshold parameter, number of videos and net bandwidth on latency. For each parameter the other parameter values are kept constant and the simulation is run for different arrival rates.

We fix the threshold $\delta$ at $1 / 6$ th the length of the segment and run the simulation to calculate latency for four different values of $\alpha$, the ratio of multicast to patching bandwidth. To study the impact of the threshold parameter, $\delta$ we fix $\alpha=0.6$ and $\mathrm{M}$, number of videos is 10 . Similarly we for $\delta$ at $\mathrm{Ls} / 6$ and $\alpha=0.6$ we increase the number of videos to 20 to see whether the system can support more videos at a admissible

\footnotetext{
${ }^{1}$ NSFNet Backbone Topology. http://moat.nlanr.net/INFRA/NSFNET.html
} 
arrival rate. Finally we look at the impact of bandwidth enhancement on the capacity by doubling the total bandwidth.

In the second scenario we look at how the system adjusts to congestion in case of 3 and 6 distributed servers respectively. Table 5.1 lists the simulation parameters used.

Table 5.1 Simulation Parameters (Scenario 2)

\begin{tabular}{|l|l|}
\hline Video length, $L$ & $120 \mathrm{~min}$ \\
\hline The combined bandwidth at the Central Server and each sender, $B$ & $200 \mathrm{Mbps}$ \\
\hline Video coding rate, $b$ & $1.5 \mathrm{Mbps}$ \\
\hline Number of segments, $K$ & 4 \\
\hline Length of a segment, $L s$ & $30 \mathrm{~min}$ \\
\hline Patching threshold, $\delta$ & $L s / 6$ \\
\hline Multicast to patching ratio, $\alpha$ & 0.6 \\
\hline Number of patching channels & 53 \\
\hline Number of videos when $N=3$ & 10 \\
\hline Number of videos when $N=6$ & 20 \\
\hline
\end{tabular}

We are supporting 10 videos in case of 3 servers and 20 videos with 6 servers. In the case of 3 distributed servers we have $100 \%$ replication on all servers, while with 6 servers we have $50 \%$ replication i.e. first 10 videos are replicated on 3 servers and the next 10 are replicated on remaining 3 servers. 
First we consider 3 senders S1, S2 and S3 to be streaming video to the receivers. We shall look at the behavior of the system for the receivers falling in the same multicast group. Receivers R1, R2, R7 and R8 send video requests at 103, 108, 110 and 112 seconds respectively for the same video (say video 2) and so fall in the same multicast group as decided by the Central Server. There are two groups of TCP connections sharing a congestion path with $\mathrm{S} 1$ and $\mathrm{S} 3$. Congestion paths are represented with dotted lines. Link $\mathrm{X}$ is a congestion link shared with $\mathrm{R} 7$ and $\mathrm{R} 8$ and link $\mathrm{Y}$ is a congestion link shared with R1 and R2. TS1 TS20 and TS21 TS40 represent the TCP servers and TR1 TR20 and TR21 TR40 represent the TCP clients. TCP transmissions start and stop during the simulation to see if S1 and S3 adjust accordingly with the congestion.

Next we consider 6 senders S1 S6 while supporting 20 videos. Videos 1 to 10 are replicated on S1, S2 and S3 while videos 11 to 20 are replicated on S4, S5 and S6. In this case $\mathrm{R} 1$ and $\mathrm{R} 2$ request for video 12 while $\mathrm{R} 7$ and $\mathrm{R} 8$ still request video 2 . Thus $\mathrm{S} 1, \mathrm{~S} 2$ and S3 service requests from R7 and R8 while S4, S5 and S6 handle those from $\mathrm{R} 1$ and R2. R7 and R8 do not experience any congestion in this case and senders $\mathrm{S} 1 \sim \mathrm{S} 3$ continue with the initial segment schedule to stream all the segments of video 2 . R7 and R8 face congestion on link Y due to TCP connections. We shall see how the system reacts to congestion as compared to the case of 3 servers. We shall also compare the latency of the system while supporting more videos in case of 6 servers instead of 3 . In the following chapter we shall look at the obtained results in the above explained scenarios. 


\section{CHAPTER 6}

\section{RESULTS}

The simulation results are divided into two categories. First, the latency plots for varying parameter values and second, the loss rates and throughputs of the senders with congestion on different paths in the network.

\section{$\underline{6.1 \text { Latency Plots }}$}

In this section we study the impact on the startup latency for a VoD client and ability of the distributed VoD system to handle client requests. All the values obtained are in a $\pm 5 \%$ interval with a confidence level of $95 \%$. [17]

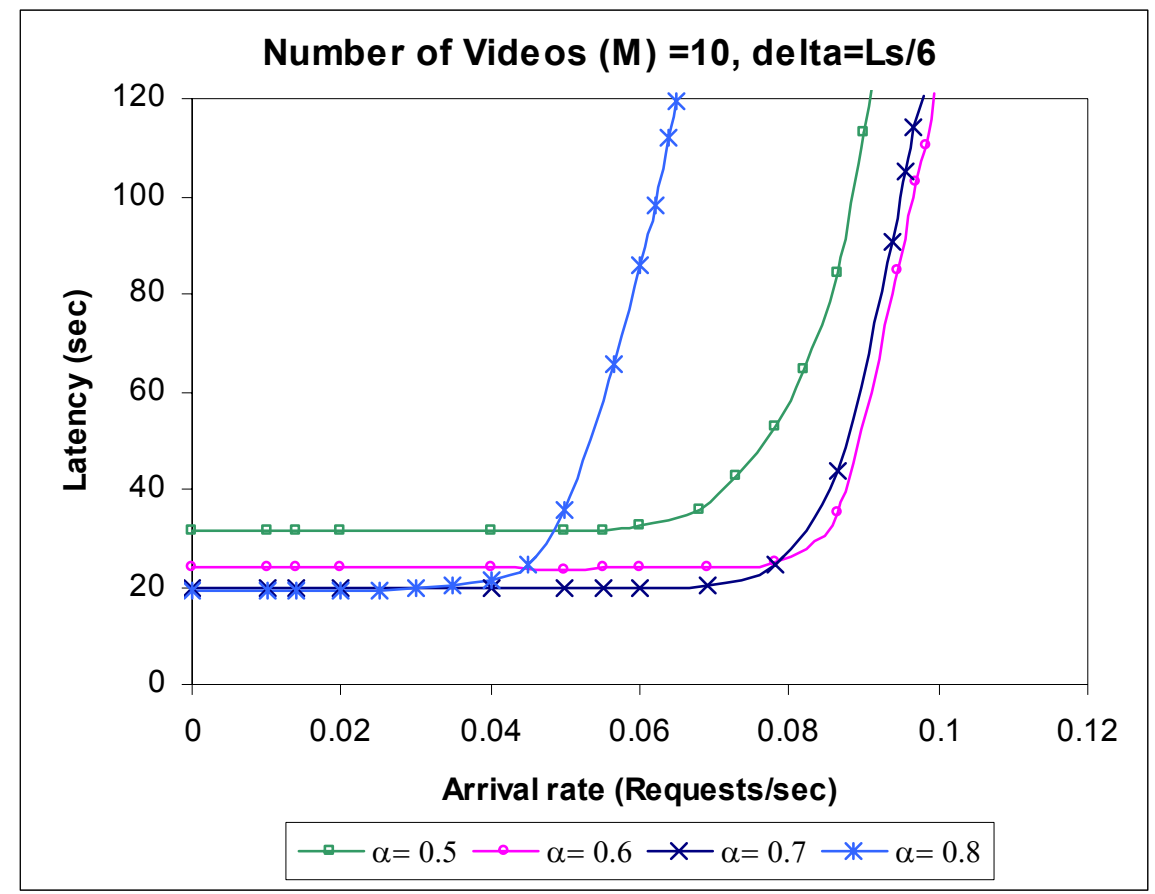

Figure 6.1 Impact of Bandwidth Partitioning on Latency 
In Figure 6.1 we show the impact that bandwidth partitioning has on latency. For a given value of $\alpha$, as we increase the arrival rate we observe that the latency stays steady till a point after which it increases rapidly. This rapid increase in the latency is due to the amount of patching bandwidth. Till the point where the latency remains steady a video request finds a patching channel available on arrival, after that it has to wait for the patching channels to be free before it is serviced. Values of $\alpha$ below 0.6 give poor performance, as the multicast component has minimum bandwidth assigned to it. Thus the latency offset which is due to the multicast delay is larger. When $\alpha$ is 0.6 we get a very good performance, as the multicast delay component is around 25 seconds and we are able to support up to 6 arrivals per minute ( 0.1 arrivals per second) with a latency of no more than 120 seconds.

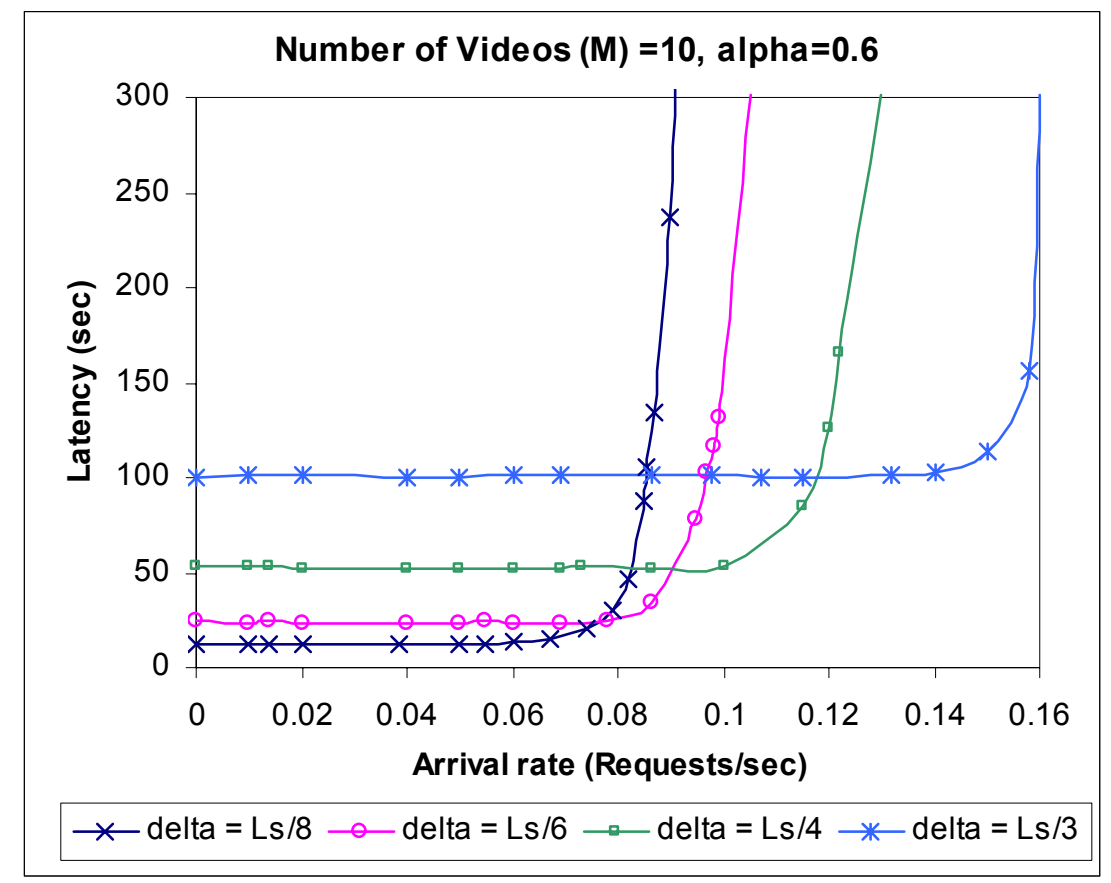

Figure 6.2 Impact of Threshold on Latency 
Next we look at the impact of $\delta$ on latency in figure 6.2. During less popular times when the request arrivals are few we can to use smaller values of $\delta(1 / 8$ th), as this would keep the latency low (less than 15 seconds). Higher values of threshold are desirable for busier times (up to 1/3rd) with a tolerable latency of 100 seconds.

The system can also support more videos with a compromise on the maximum number of supported arrivals. If the number of popular videos, $M$, is 20 as opposed to 10 then we can still support a reasonable number of arrivals, about 3 per minute. Here we can choose $\delta$ such that we can handle more requests by compromising on the latency. Figure 6.3 shows the latency plots for supporting more videos.

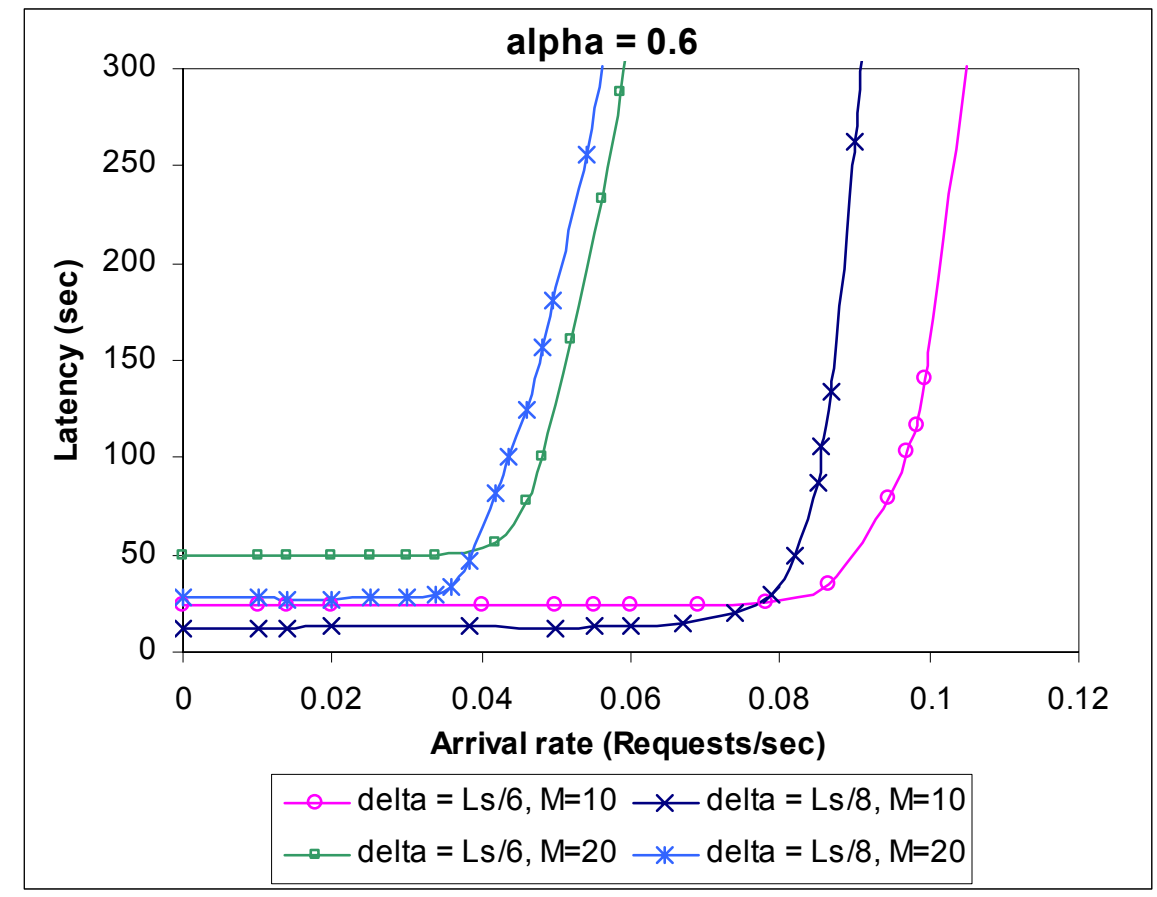

Figure 6.3 Latency when supporting more Videos

Increasing the net bandwidth also affects the latency. Figure 6.4 shows that with an increase (doubling) in bandwidth the number of requests that can be handled 
increases around five-fold ( 0.08 to 0.4 and 0.13 to 0.58 ). This indicates that bandwidth enhancements are efficiently utilized by the system to improve capacity (support more request arrivals).

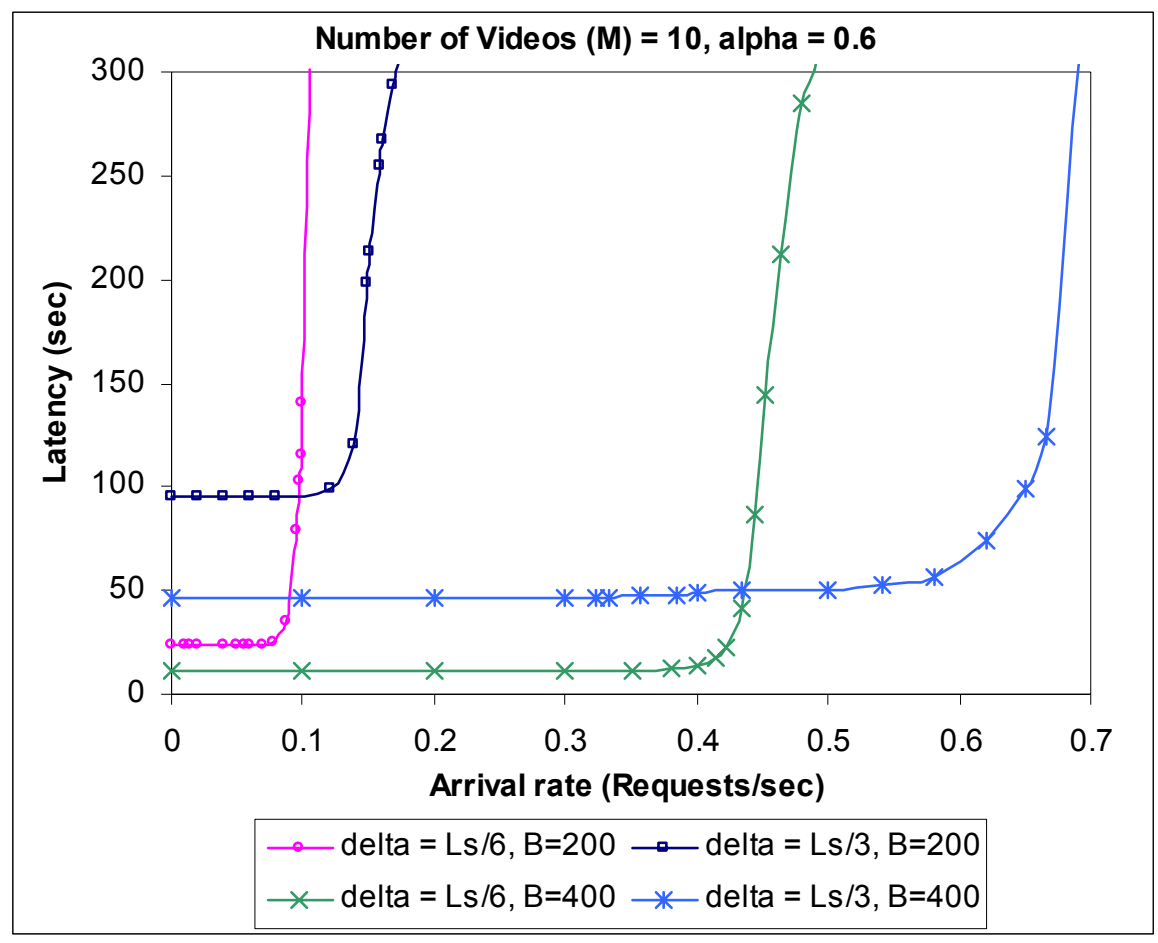

Figure 6.4 Impact of Increasing the Net Bandwidth

\subsection{Network Characteristic Plots}

We shall take a look at the graphs for loss rates and throughputs in case of 3 and 6 distributed servers respectively. In all the cases we observe that the senders adjust their sending sequence as per the network congestion characteristics to ensure continuous playback at the receivers.

Figure 6.5 is a graph of loss rates of S1, S2 and S3 measured at the R7 and R8. High loss rate for $\mathrm{S} 1$ is observed when there are TCP connections transmitting on a shared link $\mathrm{X}$ after $\mathrm{t}=455 \mathrm{~s}$ till $\mathrm{t}=487 \mathrm{~s}$. Congestion occurs on link $\mathrm{X}$ and loss rate for $\mathrm{S} 1$ 
at receivers R7 and R8 increases. After the threshold of the systolic window is reached, R7 and R8 send out control packets to notify the senders to change the segment sending sequence.

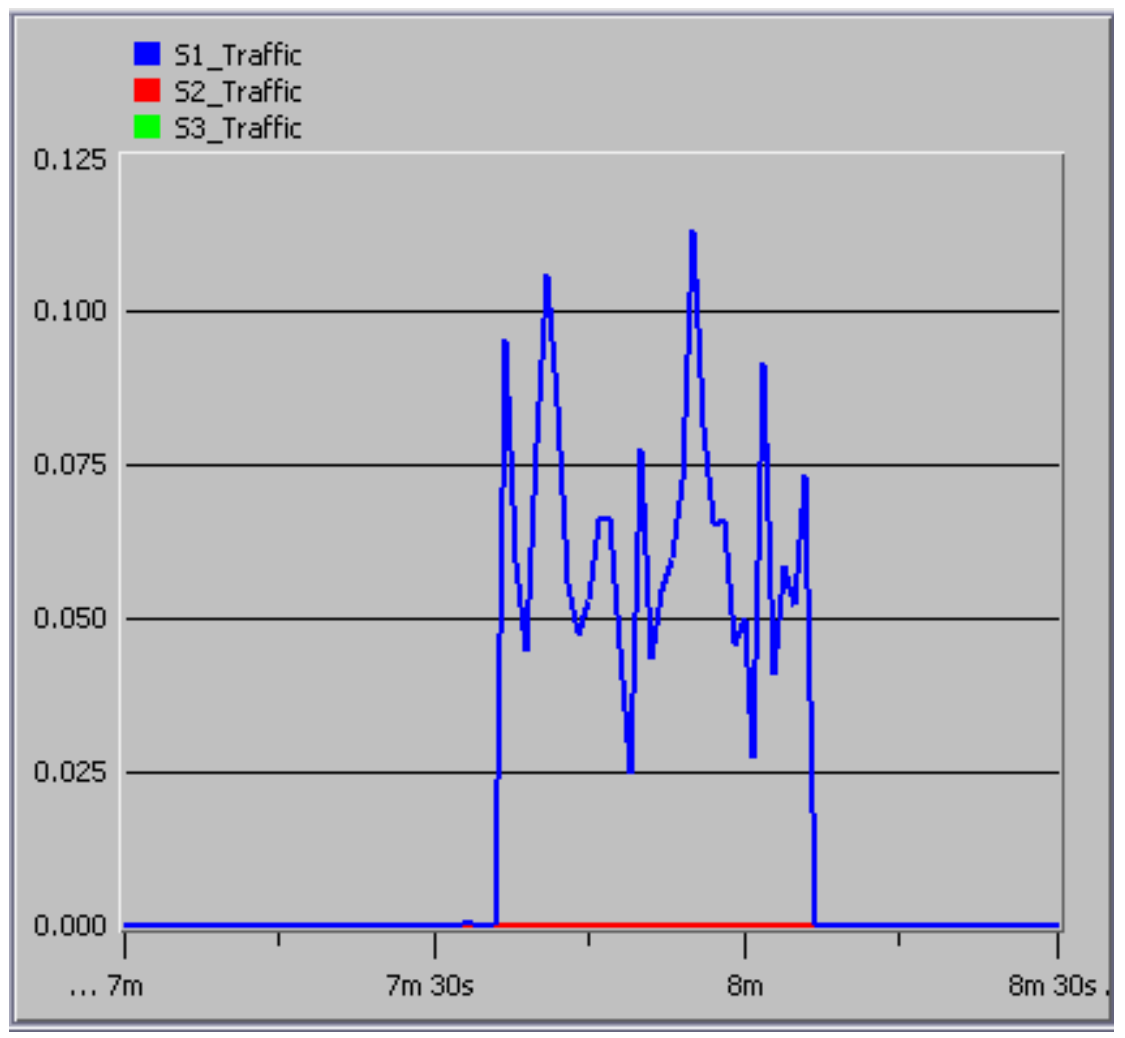

Figure 6.5 Loss rates of each sender measured at R7

In figure 6.6 and figure 6.7 we observe the throughput of S1 and S2 respectively measured at R7 during the transmission of the second segment of the video. S1 starts sending the second segment at $\mathrm{t}=451 \mathrm{~s}$. As twenty TCP sources and receivers start transmitting at $\mathrm{t}=455 \mathrm{~s}$ throughput of $\mathrm{S} 1$ begins to drop until the receivers send control packets to all the senders. Consequently S2 is selected to stream the remaining part of the second segment within the playback time of the previous $\left(1^{\text {st }}\right)$ segment. 


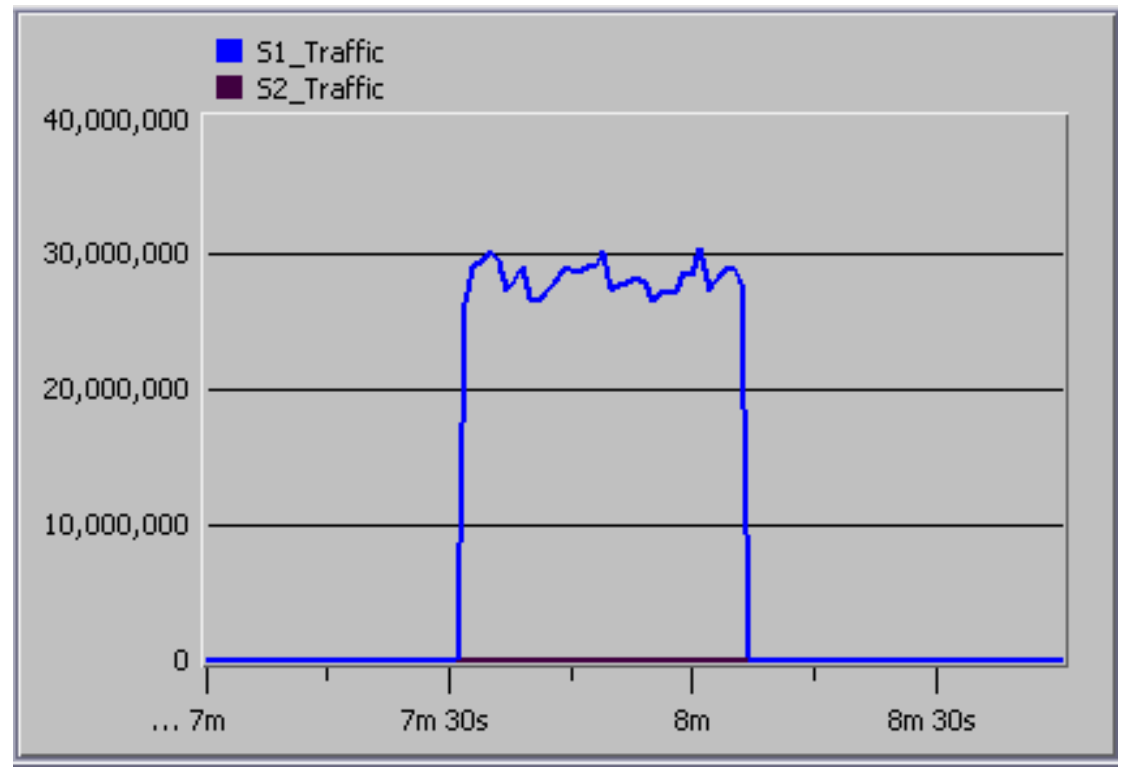

Figure 6.6 Throughputs of S1 and S2 measured at R7 (1)

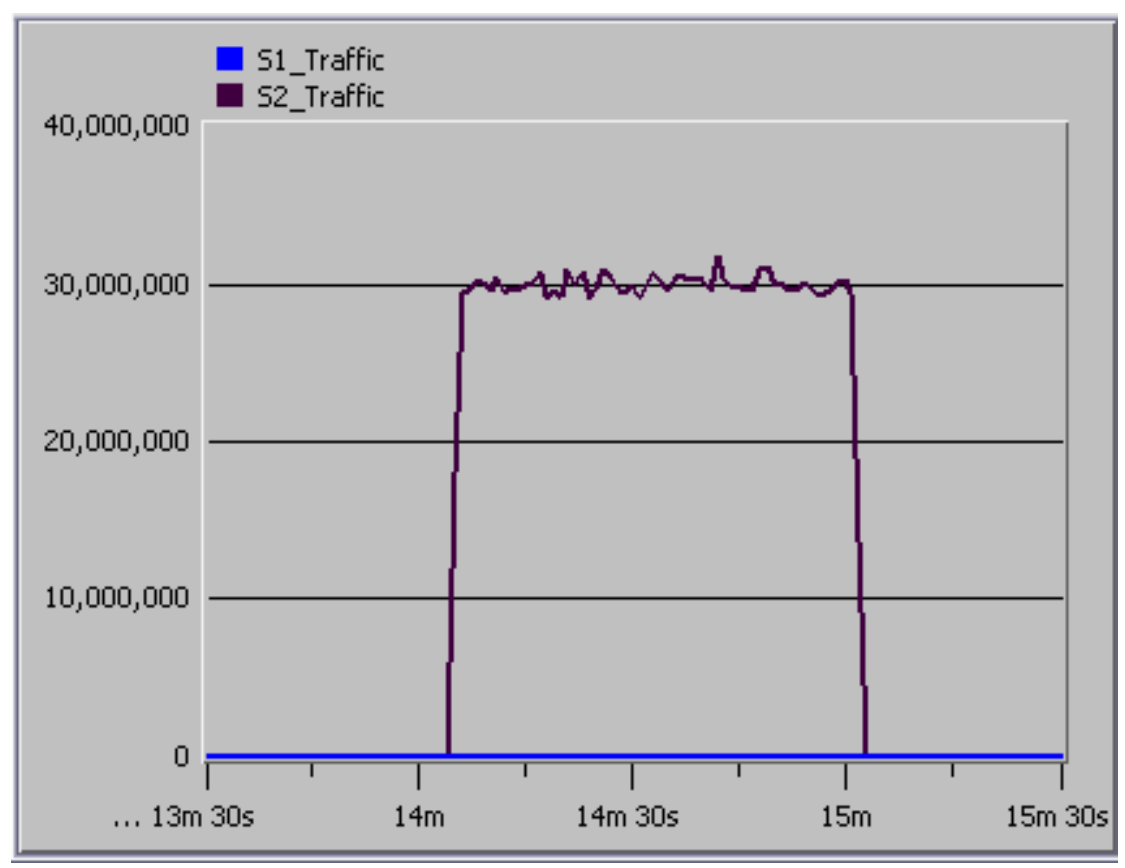

Figure 6.7 Throughputs of S1 and S2 measured at R7 (2) 
After the second segment is streamed the third segment is scheduled for streaming from S3. Figure 6.8 is a graph of loss rates of S1, S2 and S3 measured at the R1. At $\mathrm{t}=1805$ twenty TCP connections sharing a path with S3 start transmission. This causes congestion on link Y. High loss rate for S3 is observed after $\mathrm{t}=1805 \mathrm{~s}$.

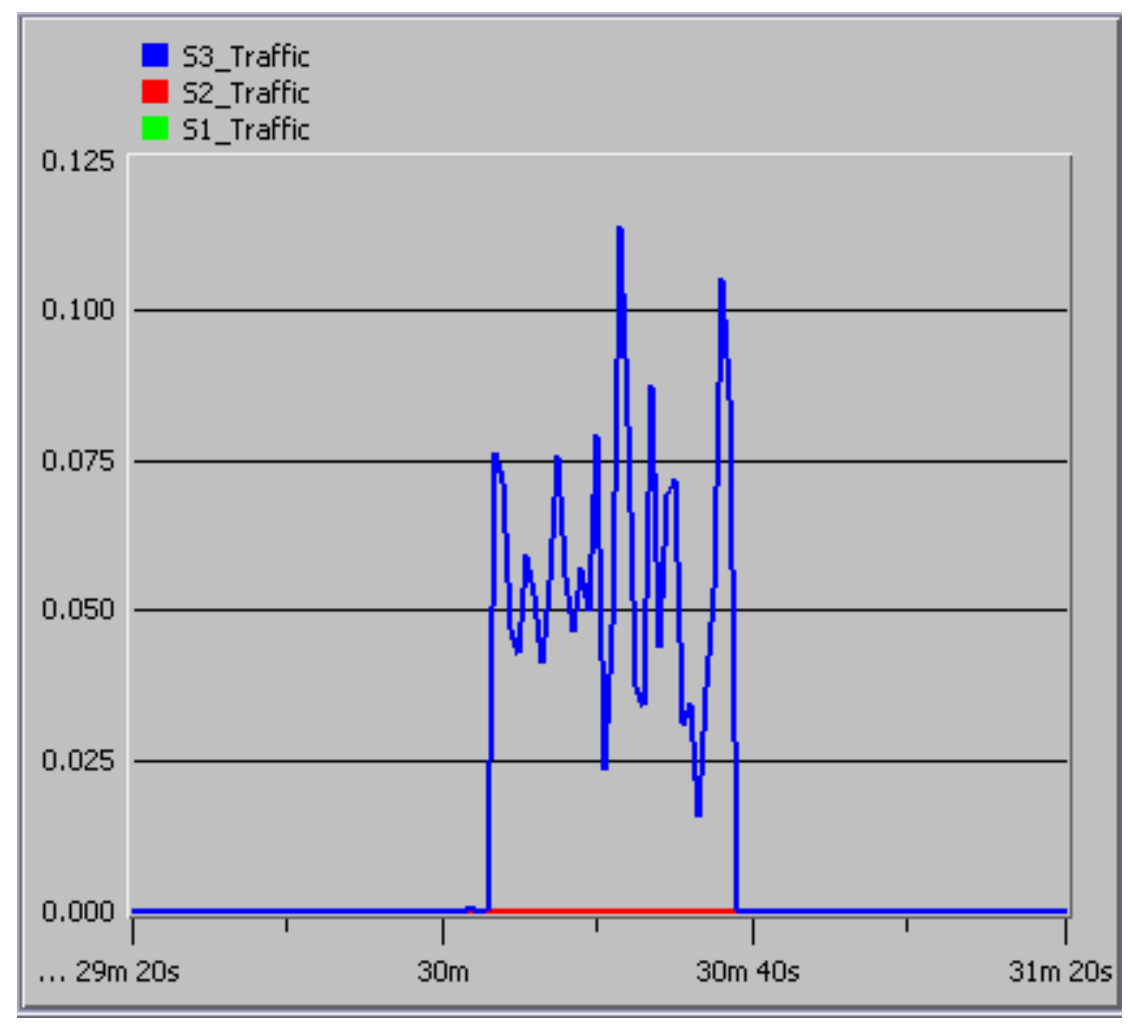

Figure 6.8 Loss rates of each sender measured at R1

Figure 6.9 shows the throughput of S3 during the transmission of the third video segment. It is seen that due to higher loss rate for S3 its throughput begins to drop beginning at $\mathrm{t}=1805 \mathrm{~s}$ till $\mathrm{t}=1838 \mathrm{~s}$; due to which $\mathrm{R} 1$ and $\mathrm{R} 2$ send control packets. As a result $\mathrm{S} 2$ is chosen to send the remaining part of the third segment as shown in figure 6.10. It is ensured that even during congestion the remaining part of the segment reaches the sender before it completes playing the previous segment. 


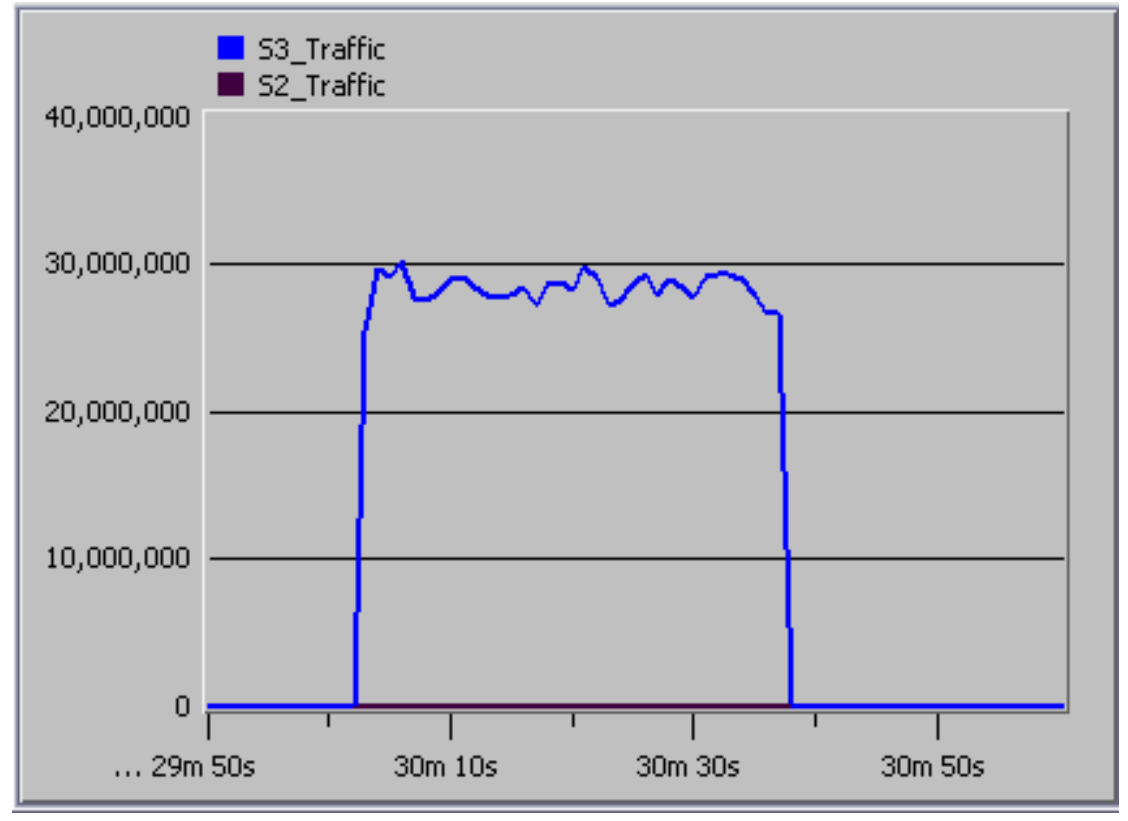

Figure 6.9 Throughputs of S2 and S3 measured at R1 (1)

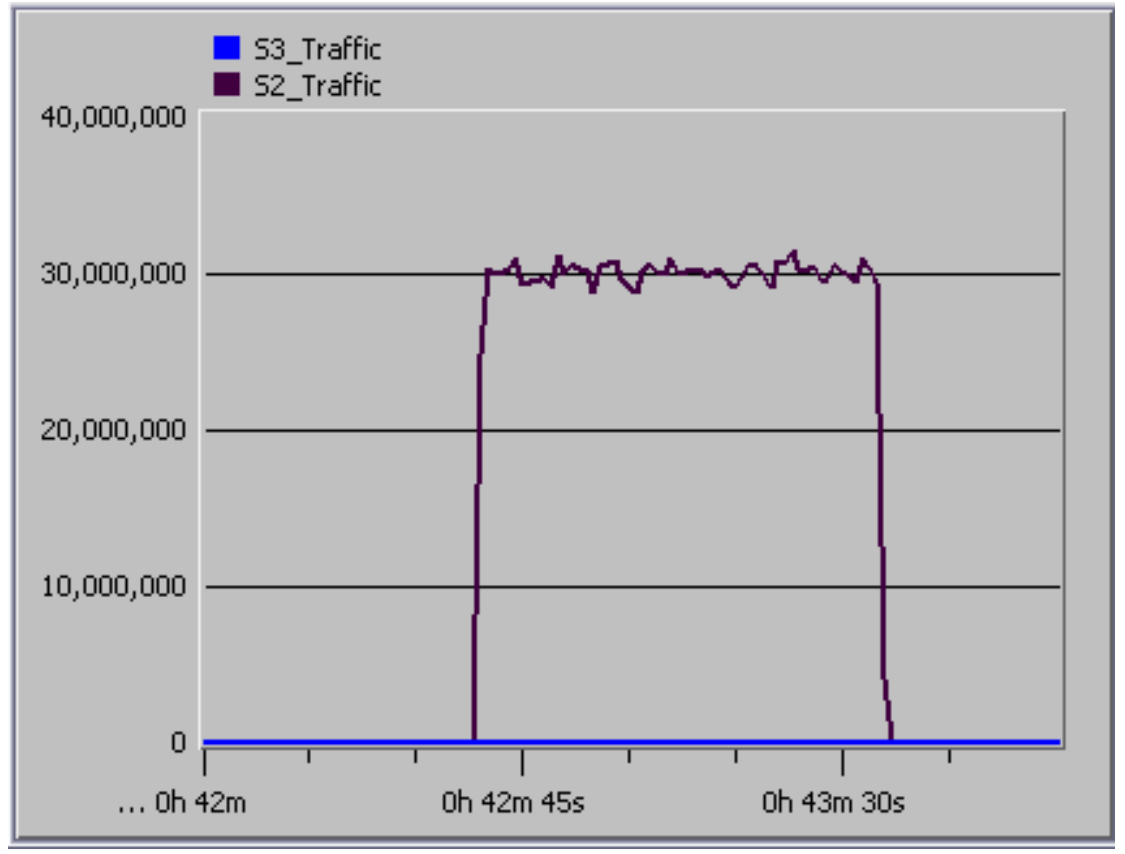

Figure 6.10 Throughputs of S2 and S3 measured at R1 (2) 
Following are the graphs plotted with 6 servers supporting 20 videos. Figure 6.11 shows the loss rates of $\mathrm{S} 4$ and $\mathrm{S} 5$ as measured at $\mathrm{R} 1$ during the transmission of the second segment of video 12 . S4 starts streaming the second segment at $\mathrm{t}=541 \mathrm{~s}$. High loss rate for $\mathrm{S} 4$ is observed due to the TCP connections on link Y after $\mathrm{t}=548 \mathrm{~s}$.

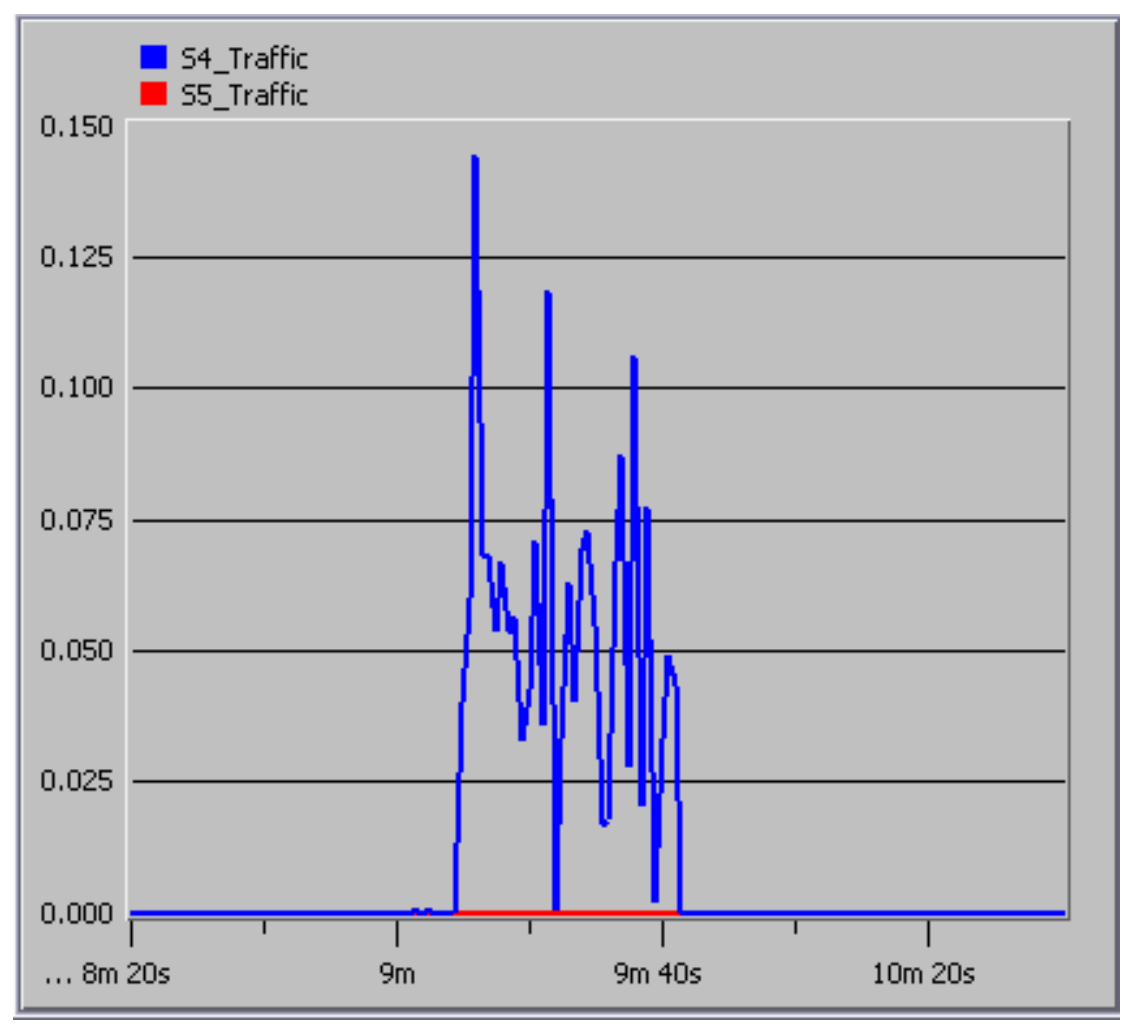

Figure 6.11 Loss rates of S4 and S5 measured at R1

Figure 6.12 and 6.13 show the throughputs of S4 and S5 as measured at R1. These show that after the loss rate for S4 rises above a certain threshold, S5 is selected to stream the remaining part of the second segment. Consecutive segments are then streamed from senders as determined by the segment partitioning algorithm. 


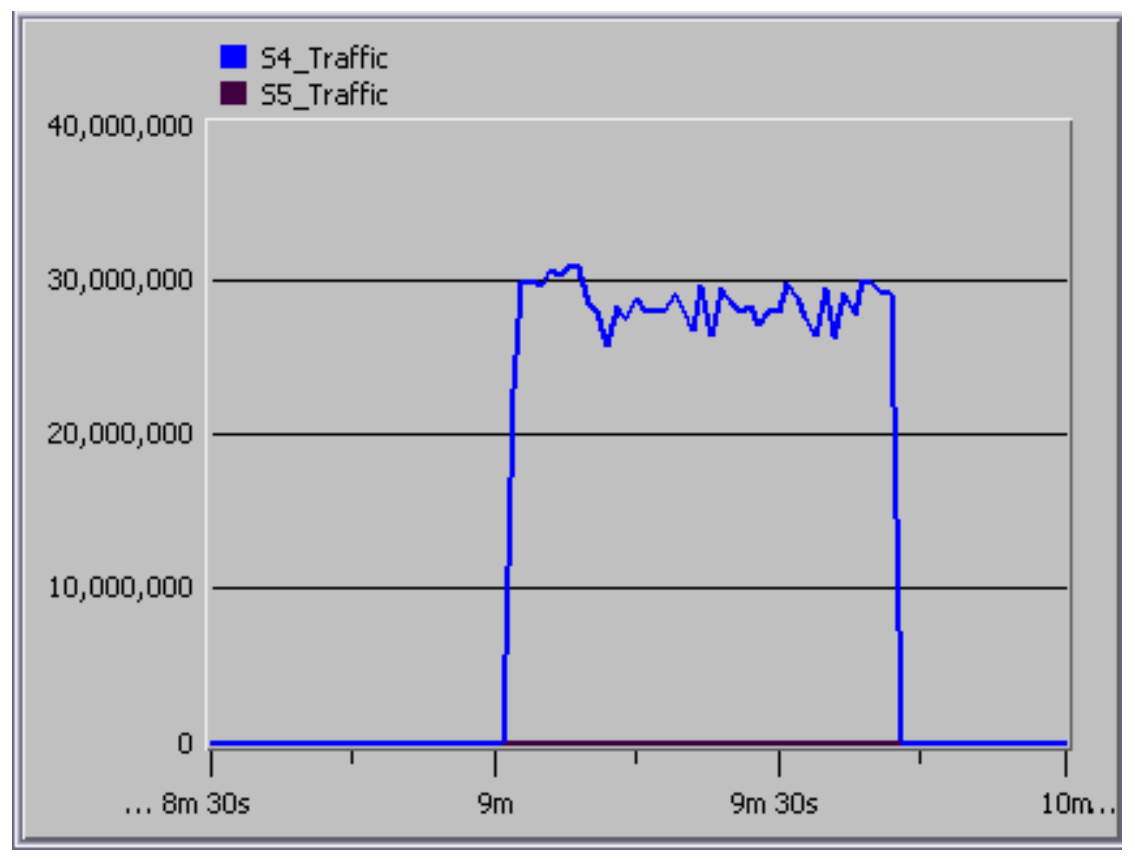

Figure 6.12 Throughputs of S4 and S5 measured at R1 (1)

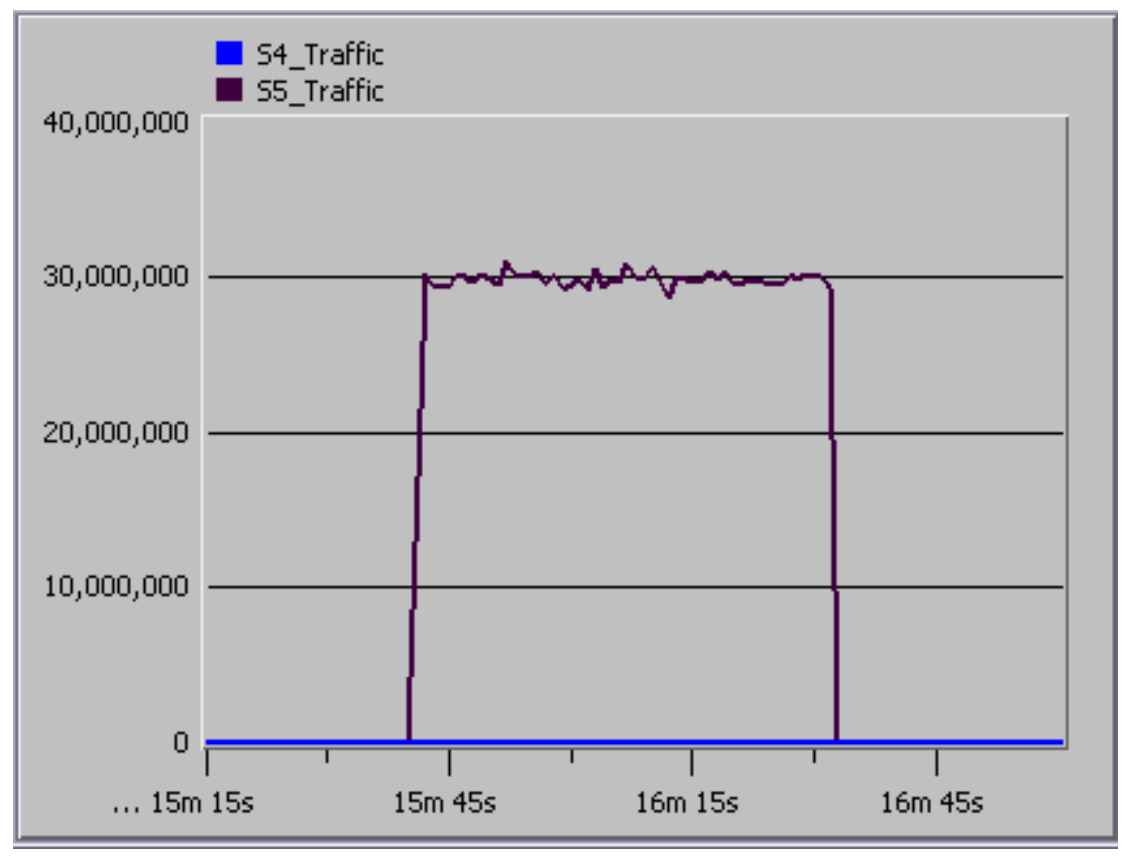

Figure 6.13 Throughputs of S4 and S5 measured at R1 (2)

Figure 6.14 shows the average latency for a request with 6 servers and 3 servers respectively supporting 20 videos. It is seen that the latency with 6 servers supporting 
20 videos is equal to that in the case of 3 servers supporting 10 videos. This is due to the difference in the percentage of replication on the servers. Thus by increasing the number of servers from 3 to 6 and varying the percentage of replication from 100 to $50 \%$ the access latency is improved (half) without affecting its distributed nature.

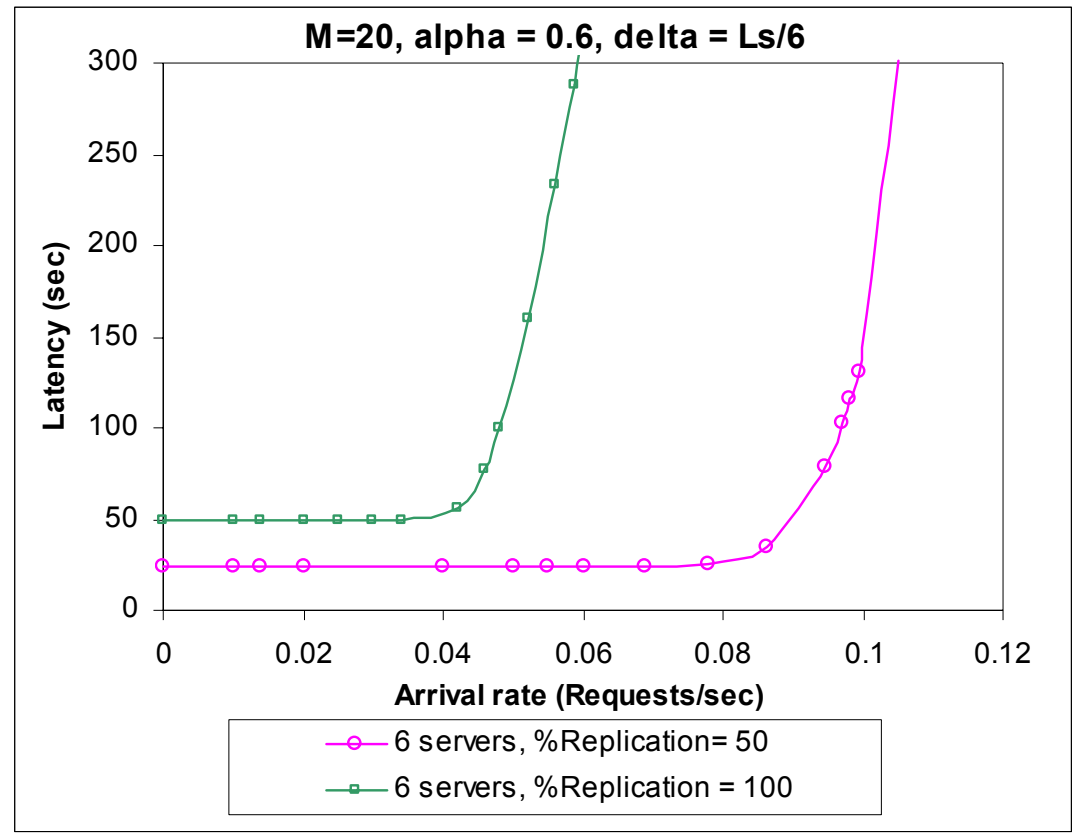

Figure 6.14 Latency: 6 Servers with Selective Placement

\section{$\underline{6.3 \text { Summary }}$}

Simulations were carried out to determine the effectiveness of Distributed Streaming for Video on Demand. We have seen the effect of various parameters on the latency provided by the system. We have also seen that the loss rate and throughput characteristics of the system while adjusting to congestion for different values of distributed servers. 


\section{CHAPTER 7}

\section{CONCLUSION}

This thesis extends existing research on Video on Demand systems by presenting a combination of two techniques, viz. DVSM and Asynchronous Hybrid mechanism for Video on Demand. Distributed streaming for Video on Demand has proposed algorithms for video streaming from multiple servers in an interleaved manner. Some of the algorithms in the base techniques were modified to suit the new Distributed Video on Demand Architecture. Roles of the sender and the receiver are modified to suit the new system while a Central Server was introduced to handle initial setup. Control packets were modified to reflect the change.

The proposed system was able to effectively incorporate the segmentation-based periodic multicast technique to effectively share network bandwidth. We were able to provide low access latencies for requests arriving in an ad hoc manner. The derived mathematical relations for latency hold good as seen by the simulation results while the characteristics of the system match well with those of DVSM with respect to adjusting to congestion.

This research can be implemented in large Video on Demand systems by increasing the number of distributed video servers and by varying the threshold and bandwidth partitioning parameters. 


\section{REFERENCES}

[1] R. Yerraballi, X. Zhao, J. Kanabar, “A New Asynchronous Hybrid Mechanism for Video on Demand". euromicro, vol. 00, no., p. 223, 29th 2003.

[2] B. Lee. "Distributed Video Streaming using Multicast", MAI 42/04, p. 1289, ISBN: 0-496-22963-0, Aug 2004.

[3] R. Zimmermann, K. Fu, C. Shahabi, D. Yao, and H. Zhu, "Yima: Design and Evaluation of a Streaming Media System for Residential Broadband Services”, Proceedings of the VLDB 2001 Workshop on Databases in Telecommunications September 2001.

[4] M. Barreiro, V. M. Gulías, J. L. Freire, and J. J. Sánchez, "An Erlang-based hierarchical distributed VoD", Proceedings of the 7th International Erlang/OTP User Conference (EUC2001). Ericsson Utvecklings AB, September 2001.

[5] S. Gonzalez, A. Navarro, J. Lopez and E. Zapata, "Two Hybrid Multicast Algorithms for Optimizing Resources in a Distributed VoD System”. Proceedings of the 10th International Multimedia Modeling Conference, January 2004, p. 145.

[6] S. Oh, S. Chung, "A Distributed VOD Server Based on VIA and Interval Cache". Proceedings of the 12th international workshop on Network and operating systems support for digital audio and video, 2002, pp. 618-625.

[7] M. Guo, M. H. Ammar, E. F. Zegura, "Selecting among replicated batching videoon-demand servers," In Proceedings of the 12th international Workshop on Network and Operating Systems Support For Digital Audio and Video, May 2002, pp. 155163. 
[8] T. P. Nguyen and A. Zakhor, "Distributed Video Streaming over the Internet," Multimedia Computing and Networking, January 2002.

[9] S. Gonzalez, A. Navarro, J. Lopez and E. Zapata, "Load sharing based on popularity in distributed Video on Demand systems", IEEE International conference on Multimedia and Expo, August 2002.

[10] T. P. Nguyen, P. Mehra and A. Zakhor, "Path diversity and bandwidth allocation for multimedia streaming", International conference on Multimedia and Expo, July 2003.

[11] T. Nguyen and A. Zakhor, "Path diversity with forward error correction (PDF) system for packet switched networks," in Proc. INFOCOM, vol. 1, 2003, pp. 663672.

[12] M. K. Bradshaw, B. Wang, L. Gao, J. Kurose, P. Shenoy, D. Towsley, S. Sen "Periodic broadcast and patching services: implementation, measurement, and analysis in an internet streaming video testbed", Proceedings of the International Conference on Multimedia 2001, Pages: 280 - 290.

[13] K. Hua, Y.Cai and S.Sheu, "Patching: A multicast technique for true video-ondemand services", ACM Multimedia'98.

[14] Streaming Video Technology, retrieved February 2005 from Sigma Designs website: http://www.sigmadesigns.com.

[15] A.O. Allen, "Probability, Statistics and Queuing Theory with Computer Science Applications", 2nd Ed., New York: Academic, 1990.

[16] OPNET Modeler, Accelerating Network R\&D.

[17] Raj Jain, "The Art of Computer Systems Performance Analysis: Techniques for Experimental Design, Measurement, Simulation, and Modeling," WileyInterscience, New York, NY, April 1991. 


\section{BIOGRAPHICAL INFORMATION}

Shraddha Rumade earned her Bachelors degree in Computer Science \& Engineering from Mumbai University, India in 2003. She began her graduate studies in Computer Science \& Engineering at the University of Texas at Arlington in August 2003 and received her M.S. in Computer Science \& Engineering in August 2005. 